OPEN ACCESS

Edited by:

Carla Sharp,

University of Houston, United States

Reviewed by:

Amanda Venta,

University of Houston, United States

Ryan Hill,

Baylor College of Medicine,

United States

*Correspondence:

Elizabeth Tianyu L

elizabeth.li@ucl.ac.uk

Specialty section:

This article was submitted to Child and Adolescent Psychiatry,

a section of the journal

Frontiers in Psychiatry

Received: 06 May 2020 Accepted: 04 November 2020 Published: 04 December 2020

Citation:

Li ET, Luyten P and Midgley N (2020) Psychological Mediators of the Association Between Childhood Emotional Abuse and Depression: A Systematic Review.

Front. Psychiatry 11:559213. doi: 10.3389/fpsyt.2020.559213

\section{Psychological Mediators of the Association Between Childhood Emotional Abuse and Depression: A Systematic Review}

\author{
Elizabeth Tianyu Li $^{1,2 *}$, Patrick Luyten ${ }^{1,2,3,4}$ and Nick Midgley ${ }^{1,2}$ \\ ${ }^{1}$ Research Department of Clinical, Educational and Health Psychology, University College London, London, United Kingdom, \\ ${ }^{2}$ Anna Freud National Centre for Children and Families, London, United Kingdom, ${ }^{3}$ Faculty of Psychology and Educational \\ Sciences, University of Leuven, Leuven, Belgium, ${ }^{4}$ Child Study Center, School of Medicine, Yale University, New Haven, CT, \\ United States
}

Background: A number of existing meta-analyses and narrative reviews have already addressed the relation between childhood adversity and depression, yet none of them has examined the specific link between emotional abuse and depression highlighted by previous research. It is no longer appropriate to regard childhood maltreatment as a unitary concept when considering its effects on subsequent depression; instead, subtypes of childhood maltreatment need to be scrutinized separately. This review addresses this significant gap by critically evaluating empirical studies examining psychological mediators of the relationship between childhood emotional abuse and subsequent depression.

Methods: A systematic search of nine electronic databases was conducted to identify eligible studies published in English between January 1980 and January 2020. Given the heterogeneous outcomes of eligible studies and the inconsistent reporting of indirect effects, a narrative synthesis, rather than a quantitative meta-analysis, was conducted. An appraisal of methodological quality was also included.

Results: We identified 34 papers, comprising 18,529 adults and 3,434 adolescents, including 888 clinical participants. Our synthesis suggests that studies on mediators in the emotional abuse-depression link have focused on five clusters of intervening variables: early maladaptive schemas, cognitive-personality variables, emotion dysregulation, interpersonal styles, and stressful negative events. Only 11 studies identified the unique contribution of emotional abuse to depression by controlling for other forms of childhood maltreatment.

Conclusions: Our findings support several routes with relative consistency (e.g., early maladaptive schemas, hopelessness, negative cognitive styles, brooding rumination, overall emotion dysregulation). Because psychological mediators function as a complex interrelated system, controlling for the interrelation between them is important. The evidence for the purported mediating role of the factors identified in this review should be considered with caution given the relative dearth of large-scale, adequately powered 
longitudinal studies. This review proposes a comprehensive multilevel theoretical framework as a basis for future research.

Keywords: depression, emotional abuse, child maltreatment, psychological mechanisms, mediator, early maladaptive schemas, cognitive-personality variables, emotion dysregulation

\section{INTRODUCTION}

Both cross-sectional retrospective studies (1-3) and longitudinal studies (4-6) have provided evidence for the role of childhood maltreatment as a precursor to depression. Systematic reviews and meta-analyses (7-9) have consistently found childhood maltreatment to be strongly associated with clinical depression across the life course, an elevated risk of recurrent and persistent depressive episodes, and a lack of response or remission during treatment. In a recent meta-analysis of 184 studies totaling 255 effect sizes, maltreated individuals were found to be $2.66-3.73$ times more likely to develop depression in adulthood, had an earlier onset of depression, and were twice more likely to develop chronic or treatment-resistant depression (10).

Whereas, the relation between childhood maltreatment and depression has been relatively well-established, much less is known about the relation between specific forms of maltreatment and depression. Historically, the more "obvious" forms of maltreatment, namely sexual and physical abuse, have attracted more attention when considering early adverse experiences associated with an increased risk of adolescent and adult depression $(11,12)$. Recently, however, researchers have started to examine particular types of childhood maltreatment as risk factors for depression, with an increasing focus on the more "silent" forms of maltreatment, such as emotional abuse. For example, studies have found that experience of parental physical abuse was least strongly associated with depressive symptoms (13), whereas self-reported childhood sexual abuse (14) and a history of emotional abuse have been more consistently associated with a wide range of psychopathology, and depression in particular (15-17). The term "childhood emotional abuse" has been used to describe exposure to spurning, terrorizing, isolating, exploiting or corrupting, and denying emotional responsiveness (18). Experiences of emotional abuse have been associated with powerful and enduring psychological sequelae, including shame, humiliation, anger, and feelings of worthlessness (19).

In a meta-analysis of 124 studies (20), emotional abuse was found to increase the risk of depression by an odds ratio of 3.06, whereas physical abuse increased the risk by an odds ratio of 1.5. In another meta-analysis of 184 studies (10), childhood emotional abuse was found to be most prominently linked to depression severity and was associated with the highest increase in the likelihood of depression in adulthood among all types of maltreatment. In addition, given that emotional abuse occurs in $90 \%$ of cases where the child is also physically or sexually abused and often precedes and persists beyond physical and sexual forms of abuse, it may be that the lasting psychological impact of different forms of abuse is largely explained by emotional abuse implicated in all forms of abuse (21). Although studies indicate that emotional abuse may play a key role in vulnerability for depression, emotional abuse is comparatively elusive, and its more "covert" nature often leads researchers and practitioners to focus on other more "tangible" forms of maltreatment in studying and treating depression $(22,23)$.

Relatively less is currently known about the potential psychological mechanisms linking emotional abuse and depression. Indeed, given that the time between exposure to abuse and onset of depression might be years or even decades, and that not all children who have experienced emotional abuse will go on to develop depression, the link between childhood emotional abuse and depression in adolescence and adulthood demands further explanation. Recent years have seen a vast increase in the number of studies investigating biological and psychosocial mechanisms addressing this issue (24). Biological mechanisms that have been investigated include genetic $(25,26)$ and neurobiological factors [e.g., impairments in HPA axis functioning; (27)]. Psychological mechanisms mainly involve maladaptive relationship and thinking styles, such as early maladaptive schemas (28), emotion dysregulation (29), hopelessness (30), insecure attachment styles (31), and interpersonal problems (32). Yet, the literature remains poorly integrated, and thus there is a need for a systematic review that has the potential to support the development of empirically supported models of depression vulnerability and maintenance and provides a platform for ensuring that psychological interventions target the most promising mechanisms, which may lead to improved treatment outcomes.

A number of existing meta-analyses and narrative reviews have already addressed the relation between adversity and depression, yet none of these meta-analyses focused on emotional abuse specifically. Braithwaite et al. (33) conducted a systematic review of longitudinal studies assessing the associations between childhood maltreatment in general and later depression. Interpersonal relationships $(31,34,35)$, cognitive vulnerabilities $(31,36)$, and behavioral difficulties $(37,38)$ were found to mediate the relation between childhood maltreatment and depression. Hoppen and Chalder (24) conducted a systematic review of 214 studies on biopsychosocial mediating and moderating variables in the relationship between childhood adversity and affective disorders. They identified various psychological and social variables as mediators between childhood adversity in general and affective disorders in adulthood, including emotional dysregulation, attentional biases, psychoform dissociation, attachment anxiety, maladaptive cognitive styles, emotion-focused coping, lack of resilience, low self-esteem, trauma-related guilt, retraumatization, maladaptive personality types, anxious arousal, chronic interpersonal stress, and low social support. Fritz et al. (39) conducted a systematic review of social, emotional, cognitive, and behavioral resilience factors that may reduce the risk of psychopathology in young people 
following childhood adversity. They found empirical support for resilience factors at the individual (e.g., high self-esteem, low rumination), family (e.g., high family cohesion, high parental involvement), and community (i.e., high social support) level that may benefit mental health in young people who have experienced childhood adversity. Most recently, Aafjes-van Doorn et al. (40) specifically examined the mediating role of cognitive factors in the relationship between childhood trauma and subsequent adult psychopathology across clinical and non-clinical populations in a systematic review of 98 empirical studies. They found that cognitive factors consistently mediated this relationship, with the vast majority of studies reporting a significant mediation effect (95\%; of which $83 \%$ was full mediation), regardless of different measures of traumatic experiences, psychopathology, and cognitive mediators. However, as noted, none of these reviews has examined the specific link between emotional abuse and depression highlighted by previous research $(10,20)$.

As a more hidden and "silent" form of childhood maltreatment, emotional abuse may be difficult to distinguish from dysfunctional parenting in general (41) and is often unrecognized and unrecorded by professionals (and wider systems) as well as laypersons (42). Regardless, empirical studies have suggested that the incidence of emotional abuse in the absence of other forms of maltreatment is more common than often assumed $(43,44)$. In the U.S. National Child Traumatic Stress Network (NCTSN) Core Data Set (CDS), emotional abuse and neglect were found to be the most prevalent form of maltreatment, where nearly one quarter $(24 \%)$ of maltreatment cases exclusively involved emotional maltreatment $(45,46)$. Not only is it the case that different forms of child maltreatment were reported to have equivalent psychiatric effects (47), but the occurrence of emotional abuse was found to be an equivalent or significantly greater predictor of 27 out of 30 negative outcomes compared with the co-occurrence of physical and sexual abuse (43). All of this points to the role that emotional abuse may play as a powerful form of childhood abuse in its own right. Given that emotional abuse constitutes a distinct form of maltreatment; misattributing its pernicious effects to other more "tangible" forms of maltreatment also has important implications for clinical practice (48). It is no longer appropriate to regard childhood maltreatment as a unitary construct; instead, subtypes of childhood maltreatment need to be scrutinized separately as well as in combination.

The current review aims to address this significant gap in the evidence base and provides a systematic review and synthesis of the empirical literature for the proposed psychological mechanisms of the relationship between childhood emotional abuse and subsequent depression, taking into consideration the robustness of the statistical mediation methods employed. Specifically, this review aims to (a) provide a comprehensive systematic review of quantitative studies investigating psychological mediators between childhood emotional abuse and depression in adolescence and in adulthood, and (b) evaluate the quality of the available evidence, including the relative strength of the statistical mediation analysis used to explain the emotional abuse-depression link.

\section{METHODS}

\section{Search Procedure}

The current review was designed in accordance with the Preferred Reporting Items for Systematic Reviews and Metaanalyses (PRISMA) guidelines $(49,50)$. Details of the protocol, including methods of the analyses and inclusion criteria, were specified in advance and documented on PROSPERO (PROSPERO registration: CRD42019127975). A systematic literature search was conducted twice by the first author, the first time in February 2019 when registering the study in PROSPERO, the second time in January 2020 to assure that more recent studies were also included. These two systematic searches identified the same set of 30 empirical studies to be included in this review and four additional studies that were published since the first review (51-54). Nine electronic databases-PsycINFO, Web of Science, MEDLINE, Scopus, and ProQuest (including PTSDpubs database, Health \& Medical Collection, Research Library, Science Database, and Social Science Database)were systematically searched using the following search terms: (depress OR internalizing OR Affective Disorder OR Mood Disorder) AND (emotional abuse OR emotional maltreatment OR verbal abuse OR emotional trauma OR psychological abuse OR child abuse OR child maltreatment). The search terms followed closely those of previously published meta-analyses of the relationship between childhood emotional abuse and depression. As different terms are used to describe mediators or mechanisms in different disciplines, we did not restrict our search to terms relating to psychological mediators. Duplicates were filtered out using EndNote Web. Examination of reference lists of eligible studies and a forward search was carried out in addition to the database search.

\section{Inclusion and Exclusion Criteria}

Eligible studies were original empirical studies that investigated the relationship between childhood emotional abuse and depression and published between 1980 and 2020 in peerreviewed journals. Eligible designs were cross-sectional, casecontrol, and prospective cohort (over any time period) studies that had assessed the association between childhood emotional abuse and depression symptoms and/or diagnosis of depression and the effect of one or more psychological mediating mechanisms on this relationship. Both a diagnosis of clinical depression and a continuous measure of depressive symptoms using scales with reported validity and reliability were considered eligible because there is evidence that depression can exist on a continuum of severity, ranging from mild, transient depressed mood states to severe, chronic forms.

Only reports that utilized mediation analysis or another suitable modeling approach to examine whether childhood emotional abuse had an indirect effect on depression outcomes via specific psychological processes were included. Our definition of psychological mechanisms was adapted from that of Harvey and Watkins (55) and considered any aspect of cognition, behavior, affective symptoms, or mood. Studies were regarded as eligible if the measures used to assess the variables under study (emotional abuse, symptoms/diagnoses, 
and putative mediating mechanisms) were described as valid and reliable. However, where studies were epidemiological in nature, measures of variables using items created for the study without further additional validation were considered eligible given that population-based studies often employ extensive surveys, necessitating briefer means of assessing each variable of interest.

Participants were adolescents or adults from any background and in any setting, including inpatients. Studies that examined the mediating mechanisms between parental emotional abuse and current depression in a child sample or emotional abuse perpetrated by peers were excluded (56). Studies that used a single item from a validated scale were also excluded, as were studies that calculated the overall effect of childhood maltreatment only and not emotional abuse specifically (57-61). Studies in languages other than English were excluded, as were single-case studies, case series, qualitative studies without a quantitative element, review articles, book chapters, dissertations, discussion papers, non-research letters, editorials, and conference abstracts.

\section{Data Extraction and Analytic Plan}

After the removal of duplicates, paper titles were analyzed by the first author. The majority of articles excluded at this stage had titles referring to either completely unrelated topics or did not meet the inclusion and exclusion criteria listed above. The abstracts of the remaining articles were then screened by the first author. All papers potentially meeting inclusion and exclusion criteria were included for full-text scrutiny. Uncertainties concerning whether a study should be included or excluded were resolved through discussions between the authors. To ensure that relevant papers were not missed, additional screening measures, consisting of conducting a citation search and screening the references of eligible studies, were conducted. The reference lists were manually checked for studies not retrieved via electronic searches.

Information about study design, sample, measures of variables, the statistical analysis used to test mediation, and outcomes of interest was extracted and integrated by using a narrative integration approach structured around the type of mediator examined (see Table 1). Given the heterogeneous outcomes of eligible studies (i.e., in terms of psychological mediators, adolescent and adult depression symptoms or diagnoses) and the inconsistent reporting of indirect effects, a narrative synthesis, rather than a quantitative meta-analysis, was conducted. Some studies did report model-related fit indices for mediation (e.g., Root Mean Square Error of Approximation) but the majority of the studies did not report mediator-related effect sizes. Hence, statistical comparison of effect sizes was not feasible.

In the present review, we grouped studies according to five types of psychological mediators in the emotional abusedepression link: these were early maladaptive schemas, cognitivepersonality variables, emotion dysregulation, interpersonal styles, and stressful negative events. These five types of mediating variables are neither necessarily independent nor mutually exclusive, but we considered them separately. In the Discussion we discuss to what extent these potential mediators might be hierarchically organized.

\section{Quality Assessment}

The first author independently evaluated study quality. Uncertainties concerning study quality were resolved through discussions between the authors. Following methodological recommendations from PRISMA (49), a component approach to quality assessment was employed. The quality of eligible papers was assessed using the Effective Public Health Practice Project tool [EPHPP; (86)] adapted to enable assessment of the specific methodological features of the primary studies pertinent to the research question under scrutiny. Our quality assessment considered the following four domains: (a) selection bias, (b) study design, (c) confounders, (d) data collection methods, and (e) withdrawals and dropouts. The analytic approach employed was also assessed and rated as "strong," "moderate," or "weak" depending on its appropriateness in terms of testing for mediation effects. Regression methods (68) where mediational effects are inferred rather than based on direct statistical observation (87) were rated as weak. A moderate rating was assigned to analyses where regression methods with additional tests of indirect effects, such as the Sobel test, had been used in addition to regression analysis. Explicit analyses estimating direct and indirect effects with bootstrapping techniques $(73,88)$ and path analysis were assigned a strong rating. Table 2 presents a summary of the quality of each eligible study.

\section{RESULTS}

\section{Characteristics of the Primary Studies}

The literature search yielded 24,563 articles. After electronically removing duplicates, the 6,290 remaining studies were screened based on title and abstract, and 6,090 articles were excluded as ineligible. The remaining 200 articles were assessed at full-text level, and 174 were excluded. An additional eight articles were included from a citation search and screening of the references of eligible studies. This resulted in 34 articles being included in the systematic review (see Figure 1). Table 1 provides an overview of the studies reviewed, including details of the research measures employed. Papers were from the USA $(n=18)$, Canada $(n=$ $2)$, Spain $(n=2)$, Germany $(n=2)$, the Netherlands $(n=2)$, Belgium $(n=2)$, Iran $(n=2)$, the UK $(n=1)$, Norway $(n$ $=1)$, Japan $(n=1)$, and Turkey $(n=1)$. Cross-sectional ( $n$ $=24)$, longitudinal $(n=9)$, and case-control $(n=1)$ designs were included.

The age range of adult samples varied substantially across eligible studies from 18 to over 65 years, young adults from 16 to 29 years, older adults from 62 to 90 years, and adolescent samples from 11 to 19 years. Thus, there was no single definition of adolescent, young adult, older adult, and adult samples in this review, and these descriptions were instead based on how the original studies defined their samples. Overall, eligible articles comprised 14,332 adults, 4,116 young adults, 81 older adults, and 3,434 adolescents, with a total of 888 clinical participants involved. Eight studies used adolescent samples, 26 studies used adult samples, nearly half of which were young adults recruited from universities $(n=11)$, and only one (51) investigated older adults. Among all eligible studies, only seven used clinical samples $(28,63,69,74,75,80,85)$. Most of the studies included 
TABLE 1 | Overview of studies included in review.

\begin{tabular}{|c|c|c|c|c|c|c|c|}
\hline $\begin{array}{l}\text { References; } \\
\text { Country }\end{array}$ & Design & Sample & $\begin{array}{l}\text { Measures } \\
\text { Childhood } \\
\text { emotional abuse }\end{array}$ & $\begin{array}{l}\text { Measures } \\
\text { Mediator(s) }\end{array}$ & $\begin{array}{l}\text { Measures } \\
\text { Depression }\end{array}$ & $\begin{array}{l}\text { Analysis of } \\
\text { mediation }\end{array}$ & Main relevant findings \\
\hline $\begin{array}{l}\text { Calvete (62); } \\
\text { Spain }\end{array}$ & Longitudinal & $\begin{array}{l}1,052 \text { adolescents aged } \\
13-17 \text { years }(499 \text { girls, } 553 \\
\text { boys, mean age }=13.43, S D \\
=1.29 \text { ) }\end{array}$ & $\begin{array}{l}\text { The 5-item } \\
\text { Psychological Abuse } \\
\text { Scale of the CTS-PC }\end{array}$ & YSQ-3 & CES-D & $\begin{array}{l}\text { Structural equation } \\
\text { modeling, } \\
\text { autoregressive } \\
\text { model, } \\
\text { bootstrapping } \\
\text { procedure (177) }\end{array}$ & $\begin{array}{l}\text { Parental emotional abuse did not predict the } \\
\text { worsening of early maladaptive schemas in the } \\
\text { follow-ups but was directly associated with } \\
\text { depressive symptoms }\end{array}$ \\
\hline $\begin{array}{l}\text { Carvalho } \\
\text { Fernando } \\
\text { et al. (63); } \\
\text { Germany }\end{array}$ & Cross-sectional & $\begin{array}{l}160 \text { adults ( } 49 \text { BPD patients, } \\
48 \text { MDD patients, } 63 \\
\text { controls) }\end{array}$ & CTQ & DERS, ERQ & $\mathrm{BDI}$ & $\begin{array}{l}\text { Multiple regression } \\
\text { analyses }\end{array}$ & $\begin{array}{l}\text { No significant impact of self-reported childhood } \\
\text { emotional abuse and emotion dysregulation on } \\
\text { depressive symptoms was found }\end{array}$ \\
\hline $\begin{array}{l}\text { Christ et al. } \\
\text { (32); The } \\
\text { Netherlands }\end{array}$ & Cross-sectional & $\begin{array}{l}276 \text { female college students } \\
\text { with a mean age of } 21.7 \\
\text { years }(S D=2.38)\end{array}$ & CTQ-SF & DERS, IIP-32 & $\begin{array}{l}\text { Quick Inventory of } \\
\text { Depressive } \\
\text { Symptoms }\end{array}$ & $\begin{array}{l}\text { Multiple regression } \\
\text { analyses, the } \\
\text { PROCESS tool } \\
\text { (88) }\end{array}$ & $\begin{array}{l}\text { The effect of childhood emotional abuse on } \\
\text { depressive symptoms was mediated by } \\
\text { emotion dysregulation and the following } \\
\text { domains of interpersonal problems: } \\
\text { cold/distant and domineering/controlling }\end{array}$ \\
\hline $\begin{array}{l}\text { Coates and } \\
\text { Messman- } \\
\text { Moore (64); } \\
\text { USA }\end{array}$ & Cross-sectional & $\begin{array}{l}771 \text { female undergraduate } \\
\text { students between the ages } \\
\text { of } 18 \text { and } 25 \text { with a mean } \\
\text { age of } 18.78(S D=1.02)\end{array}$ & $\begin{array}{l}\text { The Computer } \\
\text { Assisted } \\
\text { Maltreatment } \\
\text { Inventory }\end{array}$ & DERS, YSQ-SF & TSI & $\begin{array}{l}\text { Structural } \\
\text { equation modeling }\end{array}$ & $\begin{array}{l}\text { Both emotion dysregulation and negative } \\
\text { internalized beliefs significantly mediated the } \\
\text { link between childhood psychological } \\
\text { maltreatment and depressive symptoms, } \\
\text { accounting for } 68 \% \text { of the variance in } \\
\text { symptomatology }\end{array}$ \\
\hline $\begin{array}{l}\text { Courtney } \\
\text { et al. (65); } \\
\text { USA }\end{array}$ & Cross-sectional & $\begin{array}{l}195 \text { adolescent primary care } \\
\text { patients }(21.5 \% \text { male, } 78.5 \% \\
\text { female), aged } 15-18 \text { years } \\
(M=16.30, S D=1.07)\end{array}$ & $\begin{array}{l}\text { Three items adapted } \\
\text { from CTQ that } \\
\text { directly assess } \\
\text { verbal and nonverbal } \\
\text { emotional abuse }\end{array}$ & BHS & $\mathrm{BDI}$ & $\begin{array}{l}\text { Multiple regression } \\
\text { analyses }\end{array}$ & $\begin{array}{l}\text { Hopelessness partially mediated the } \\
\text { associations of emotional abuse with risk for } \\
\text { depressive symptoms. Hopelessness } \\
\text { accounted for } 69.8 \% \text { of the variance in the } \\
\text { association between emotional abuse and } \\
\text { depressive symptoms }\end{array}$ \\
\hline $\begin{array}{l}\text { Courtney } \\
\text { et al. (30); } \\
\text { USA }\end{array}$ & Longitudinal & $\begin{array}{l}92 \text { adolescent primary care } \\
\text { patients ( } 18.5 \% \text { boys, } 81.5 \% \\
\text { girls) between } 15 \text { and } 18 \\
\text { years of age }(M=17.48, S D \\
=1.27 \text { ) }\end{array}$ & $\begin{array}{l}\text { Three items adapted } \\
\text { from CTQ that } \\
\text { directly assess } \\
\text { verbal and nonverbal } \\
\text { emotional abuse }\end{array}$ & BHS & BDI-II & $\begin{array}{l}\text { Multiple regression } \\
\text { analyses }\end{array}$ & $\begin{array}{l}\text { Hopelessness partially mediated the } \\
\text { association between } \mathrm{T} 1 \text { emotional abuse and } \\
\mathrm{T} 2 \text { depressive symptoms. Hopelessness } \\
\text { accounted for } 87.3 \% \text { of the variance in this } \\
\text { association }\end{array}$ \\
\hline $\begin{array}{l}\text { Crow et al. } \\
\text { (29); USA }\end{array}$ & Cross-sectional & $\begin{array}{l}3,902 \text { adults aged } 18-81 \\
\text { years }(M=39.34, S D= \\
13.76,68.9 \% \text { women, } \\
92.7 \% \text { African American })\end{array}$ & CTQ & EDS & BDI-II & (73) & $\begin{array}{l}\text { Emotion dysregulation mediated the } \\
\text { relationship between childhood emotional } \\
\text { abuse and later depression }\end{array}$ \\
\hline $\begin{array}{l}\text { Gibb et al. } \\
\text { (66); USA }\end{array}$ & Longitudinal & $\begin{array}{l}297 \text { university students ( } 145 \\
\text { high cognitive risk, mean age } \\
=18.92,68.3 \% \text { female; } 152 \\
\text { low cognitive risk, mean age } \\
=19.28,68.4 \% \text { female) }\end{array}$ & $\begin{array}{l}\text { The maltreatment } \\
\text { subscale of the LEQ }\end{array}$ & CSQ, DAS & BDI, BHS, SADS & $\begin{array}{l}\text { Hierarchical } \\
\text { regression analysis }\end{array}$ & $\begin{array}{l}\text { Cognitive risk fully mediated the relation } \\
\text { between reported levels of childhood emotional } \\
\text { maltreatment and non-endogenous major } \\
\text { depression as well as hopelessness depression }\end{array}$ \\
\hline
\end{tabular}


TABLE 1 | Continued

\begin{tabular}{|c|c|c|c|c|c|c|c|}
\hline $\begin{array}{l}\text { References; } \\
\text { Country }\end{array}$ & Design & Sample & $\begin{array}{l}\text { Measures } \\
\text { Childhood } \\
\text { emotional abuse }\end{array}$ & $\begin{array}{l}\text { Measures } \\
\text { Mediator(s) }\end{array}$ & $\begin{array}{l}\text { Measures } \\
\text { Depression }\end{array}$ & $\begin{array}{l}\text { Analysis of } \\
\text { mediation }\end{array}$ & Main relevant findings \\
\hline $\begin{array}{l}\text { Gibb et al. } \\
\text { (67); USA }\end{array}$ & Cross-sectional & $\begin{array}{l}220 \text { undergraduates aged } \\
17-26 \text { years }(164 \text { women, } 56 \\
\text { men, mean age }=18.79, S D \\
=1.40)\end{array}$ & LEQ & $\mathrm{BHS}$ & $\mathrm{HDSQ}$ & $\begin{array}{l}\text { Path analyses } \\
\text { using AMOS } 4.0\end{array}$ & $\begin{array}{l}\text { Hopelessness partially mediated the relation } \\
\text { between childhood emotional maltreatment } \\
\text { and symptoms of hopelessness depression }\end{array}$ \\
\hline \multirow[t]{2}{*}{$\begin{array}{l}\text { Hankin (31); } \\
\text { USA }\end{array}$} & $\begin{array}{l}\text { Study 1: } \\
\text { Longitudinal (10 } \\
\text { weeks) }\end{array}$ & $\begin{array}{l}\text { Study 1: } 652 \text { undergraduate } \\
\text { students aged 17-23 years } \\
\text { (mean }=18.7, S D=0.96 \\
210 \text { males) }\end{array}$ & Study 1: LEQ & $\begin{array}{l}\text { Study 1: CSQ, } \\
\text { NLEQ, AAQ }\end{array}$ & Study 1: BDI, MASQ & $\begin{array}{l}\text { Study 1: Path } \\
\text { analyses (68), } \\
\text { univariate and } \\
\text { multivariate } \\
\text { mediational } \\
\text { analyses (178) }\end{array}$ & $\begin{array}{l}\text { Study 1: An insecure attachment style and } \\
\text { negative life events almost completely } \\
\text { mediated the association between childhood } \\
\text { emotional abuse and later depressive } \\
\text { symptoms, while a negative cognitive style was } \\
\text { minimized in the multivariate mediational model }\end{array}$ \\
\hline & $\begin{array}{l}\text { Study 2: } \\
\text { Longitudinal (2 } \\
\text { years) }\end{array}$ & $\begin{array}{l}\text { Study 2: } 75 \text { undergraduate } \\
\text { students aged } 18-23 \text { years } \\
\text { (mean }=18.6, S D=0.84 \\
34 \text { males) }\end{array}$ & Study 2: CECA & $\begin{array}{l}\text { Study 2: CSQ, } \\
\text { NLEQ, AAQ }\end{array}$ & Study 2: BDI, MASQ & $\begin{array}{l}\text { Study 2: Path } \\
\text { analyses (68), } \\
\text { univariate and } \\
\text { multivariate } \\
\text { mediational } \\
\text { analyses (178) }\end{array}$ & $\begin{array}{l}\text { Study 2: Only an insecure attachment style and } \\
\text { negative life events remained partially } \\
\text { accounted for the association between } \\
\text { childhood emotional abuse and later } \\
\text { depressive symptoms in the multivariate } \\
\text { mediational model }\end{array}$ \\
\hline $\begin{array}{l}\text { Hayashi et al. } \\
\text { (69); Japan }\end{array}$ & Cross-sectional & $\begin{array}{l}113 \text { untreated, newly } \\
\text { diagnosed MDD patients } \\
\text { aged } 25-75 \text { years ( } 58 \\
\text { women and } 55 \text { men, mean } \\
\text { age } 41.91 \text { years, } S D= \\
11.20 \text { ) }\end{array}$ & CATS & LES, NEO-FFI & BDI-II & $\begin{array}{l}\text { Structural } \\
\text { equation modeling }\end{array}$ & $\begin{array}{l}\text { Childhood emotional abuse predicted the } \\
\text { severity of depression indirectly through the } \\
\text { mediation of personality: Neuroticism, } \\
\text { Extroversion, and Conscientiousness. The } \\
\text { negative life change was affected by childhood } \\
\text { emotional abuse but did not predict the } \\
\text { severity of depression }\end{array}$ \\
\hline $\begin{array}{l}\text { Jessar et al. } \\
\text { (70); USA }\end{array}$ & Longitudinal & $\begin{array}{l}204 \text { early adolescents }(52 \% \\
\text { African American, } 54 \% \\
\text { female, mean age }=12.85 \\
\text { years) }\end{array}$ & $\begin{array}{l}\text { The emotional abuse } \\
\text { and emotional } \\
\text { neglect subscales of } \\
\text { the CTQ }\end{array}$ & $\mathrm{ECQ}$ & $\mathrm{CDI}$ & (73) & $\begin{array}{l}\text { Emotional abuse did not significantly predict } \\
\text { deficits in emotional clarity but did predict } \\
\text { increases in depressive symptoms. Deficits in } \\
\text { emotional clarity only mediated the relationship } \\
\text { between emotional neglect and increases in } \\
\text { depressive symptoms }\end{array}$ \\
\hline $\begin{array}{l}\text { Kaysen et al. } \\
\text { (71); USA }\end{array}$ & Cross-sectional & $\begin{array}{l}206 \text { adult women who had } \\
\text { been recently raped }(N= \\
133) \text { or physically assaulted } \\
(N=73) \text { (mean age }=31.21 \\
\text { years, } S D=8.58, \text { range }= \\
18-57)\end{array}$ & $H V Q$ & PBRS & BDI, SCID-MDD & (68) & $\begin{array}{l}\text { No evidence for associations of childhood } \\
\text { emotional abuse with either maladaptive } \\
\text { cognitions or depression }\end{array}$ \\
\hline $\begin{array}{l}\text { Khosravani } \\
\text { et al. (52); Iran }\end{array}$ & Cross-sectional & $\begin{array}{l}350 \text { males (age range }= \\
18-61 \text { years, } M=32.3 \text { ) }\end{array}$ & CTQ-SF & DERS & BDI-II & $\begin{array}{l}\text { Structural equation } \\
\text { models (SEM), } \\
\text { bootstrapping }\end{array}$ & $\begin{array}{l}\text { A direct effect of emotional abuse on } \\
\text { depressive symptoms and an indirect effect via } \\
\text { emotion dysregulation }\end{array}$ \\
\hline
\end{tabular}


TABLE 1 | Continued

\begin{tabular}{|c|c|c|c|c|c|c|c|}
\hline $\begin{array}{l}\text { References; } \\
\text { Country }\end{array}$ & Design & Sample & $\begin{array}{l}\text { Measures } \\
\text { Childhood } \\
\text { emotional abuse }\end{array}$ & $\begin{array}{l}\text { Measures } \\
\text { Mediator(s) }\end{array}$ & $\begin{array}{l}\text { Measures } \\
\text { Depression }\end{array}$ & $\begin{array}{l}\text { Analysis of } \\
\text { mediation }\end{array}$ & Main relevant findings \\
\hline $\begin{array}{l}\text { Krause et al. } \\
\text { (72); USA }\end{array}$ & Cross-sectional & $\begin{array}{l}127 \text { adults aged } 18-30 \text { years } \\
\text { (mean age }=20, S D=2.76, \\
78 \text { women, } 76 \% \text { Caucasian) }\end{array}$ & $\begin{array}{l}\text { Psychological Abuse } \\
\text { Scale (179) }\end{array}$ & $\begin{array}{l}\text { WBSI, AEQ, CSQ, } \\
\text { the } 11 \text {-item avoidant } \\
\text { reactions subscale } \\
\text { of the Impact of } \\
\text { Event Scale }\end{array}$ & $\mathrm{BDI}$ & $\begin{array}{l}\text { Structural equation } \\
\text { modeling, AMOS }\end{array}$ & $\begin{array}{l}\text { Chronic emotional inhibition (including } \\
\text { ambivalent expression, thought suppress, } \\
\text { current and chronic avoid) fully mediated the } \\
\text { relationship between childhood emotional } \\
\text { invalidation (including emotional abuse) and } \\
\text { depressive symptoms in adulthood }\end{array}$ \\
\hline $\begin{array}{l}\text { Li et al. (54); } \\
\text { UK }\end{array}$ & Cross-sectional & $\begin{array}{l}205 \text { adults }(80.5 \% \text { female; } \\
\text { mean age }=28.2, S D= \\
10.86)\end{array}$ & $\begin{array}{l}7 \text {-item Childhood } \\
\text { Emotional Abuse } \\
\text { Scale created and } \\
\text { validated by Kent } \\
\text { and Waller (180) }\end{array}$ & $\mathrm{RFQ}$ & $\begin{array}{l}7 \text { items of the } \\
\text { Depression subscale } \\
\text { in the DASS- } 21\end{array}$ & $\begin{array}{l}\text { Multiple regression } \\
\text { analyses, } \\
\text { PROCESS macro } \\
\text { (88) }\end{array}$ & $\begin{array}{l}\text { Emotional abuse continued to exert a } \\
\text { significant effect on adulthood depression after } \\
\text { controlling for other forms of childhood } \\
\text { maltreatment and mentalizing incapacity. A } \\
\text { mediation effect between childhood emotional } \\
\text { abuse and adulthood depression symptoms via } \\
\text { mentalizing incapacity (both hypermentalizing } \\
\text { and hypomentalizing) was established }\end{array}$ \\
\hline $\begin{array}{l}\text { Lumley and } \\
\text { Harkness } \\
\text { (28); Canada }\end{array}$ & Cross-sectional & $\begin{array}{l}76 \text { depressed adolescents } \\
\text { meeting DSM-IV criteria for a } \\
\text { current episode of a } \\
\text { non-bipolar mood disorder, } \\
\text { aged } 13-19 \text { years ( } 24 \text { boys, } \\
52 \text { girls) }\end{array}$ & $\begin{array}{l}\text { The teenage version } \\
\text { of the CECA }\end{array}$ & YSQ & $\begin{array}{l}\text { MASQ, BDI-II, The } \\
\text { child and adolescent } \\
\text { version of the SADS }\end{array}$ & $(68,73)$ & $\begin{array}{l}\text { A different set of early maladaptive schemas } \\
\text { with contents, such as social isolation and } \\
\text { self-sacrifice, mediated the relation of } \\
\text { emotional maltreatment to anhedonic } \\
\text { depression. But no specificity was found in the } \\
\text { relation between emotional maltreatment and } \\
\text { anhedonic depression }\end{array}$ \\
\hline $\begin{array}{l}\text { Lumley and } \\
\text { Harkness } \\
\text { (28); Canada }\end{array}$ & Cross-sectional & $\begin{array}{l}91 \text { young adults aged } 17-21 \\
\text { years }(21 \text { men, } 70 \text { women, } \\
\text { mean age }=18.10, S D= \\
0.84)\end{array}$ & CECA.Q & $\begin{array}{l}\text { YSQ, The Modified- } \\
\text { Psychological } \\
\text { Distance Scaling } \\
\text { Task }\end{array}$ & $\begin{array}{l}\text { BDI, the Mood } \\
\text { Disorder Module of } \\
\text { the SCID }\end{array}$ & (68), Sobel test & $\begin{array}{l}\text { Depressotypic cognitive organization (i.e., } \\
\text { tightly connected negative schema } \\
\text { organization and loosely connected positive } \\
\text { schema organization) mediated the relation } \\
\text { between childhood emotional maltreatment } \\
\text { and young adult depression }\end{array}$ \\
\hline $\begin{array}{l}\text { Maciejewski } \\
\text { and Mazure } \\
\text { (74); USA }\end{array}$ & Case-control & $\begin{array}{l}50 \text { adults ( } 25 \text { cases, } 25 \\
\text { controls) aged } 23-65 \text { years } \\
(M=39.6, S D=10.3,24 \\
\text { women, } 42 \text { European } \\
\text { American descent) }\end{array}$ & ETI & $\begin{array}{l}\text { The Fear of Criticism } \\
\text { and Rejection } \\
\text { subscale in Beck's } \\
\text { Sociotropy- } \\
\text { Autonomy } \\
\text { Scale }\end{array}$ & $\begin{array}{l}\text { SCID, CES-D, } \\
\text { HRSD, the University } \\
\text { of Michigan version } \\
\text { of CIDI }\end{array}$ & $\begin{array}{l}\text { Multiple logistic } \\
\text { regression, } \\
\text { multivariate } \\
\text { analysis of } \\
\text { covariance } \\
\text { (MANCOVA) }\end{array}$ & $\begin{array}{l}\text { Fear of criticism and rejection mediated the } \\
\text { association between childhood emotional } \\
\text { abuse and adult onset of major depression }\end{array}$ \\
\hline $\begin{array}{l}\text { O'Mahen } \\
\text { et al. (21); } \\
\text { USA }\end{array}$ & Cross-sectional & $\begin{array}{l}140 \text { pregnant women, age } \\
18 \text { or older, } 24 \text { or more } \\
\text { weeks pregnant }\end{array}$ & CTQ & RRS, BADS & SCID-I, EPDS, BDI-\|I & $\begin{array}{l}\text { Nested path } \\
\text { models in AMOS } \\
18.0, \\
\text { bootstrapping } \\
\text { procedure (177) }\end{array}$ & $\begin{array}{l}\text { Brooding partially mediated the relationship } \\
\text { between childhood emotional abuse and } \\
\text { depressive symptoms, whereas behavioral } \\
\text { avoidance was not significantly correlated with } \\
\text { childhood emotional abuse }\end{array}$ \\
\hline
\end{tabular}$$
\text { emo }
$$

depression. But no specificity was found in the anhedonic depression

Distance Scaling

The Fear of Criticism

and Rejection

Sociotropy

Scale

RRS, BADS

procedure (177) 


\begin{tabular}{|c|c|c|c|c|c|c|c|}
\hline $\begin{array}{l}\text { References; } \\
\text { Country }\end{array}$ & Design & Sample & $\begin{array}{l}\text { Measures } \\
\text { Childhood } \\
\text { emotional abuse }\end{array}$ & $\begin{array}{l}\text { Measures } \\
\text { Mediator(s) }\end{array}$ & $\begin{array}{l}\text { Measures } \\
\text { Depression }\end{array}$ & $\begin{array}{l}\text { Analysis of } \\
\text { mediation }\end{array}$ & Main relevant findings \\
\hline $\begin{array}{l}\text { Østefjells } \\
\text { et al. (75); } \\
\text { Norway }\end{array}$ & Cross-sectional & $\begin{array}{l}261 \text { patients aged } 18-65 \\
\text { years with psychotic or } \\
\text { bipolar disorders }\end{array}$ & CTQ-SF & $\begin{array}{l}\text { The subscale } \\
\text { measuring negative } \\
\text { beliefs about the } \\
\text { uncontrollability and } \\
\text { danger of thoughts } \\
\text { of the } \\
\text { Metacognitions } \\
\text { Questionnaire-30 } \\
\text { items }\end{array}$ & $\begin{array}{l}\text { Positive and } \\
\text { Negative Syndrome } \\
\text { Scale Score }\end{array}$ & $\begin{array}{l}\text { Ordinary } \\
\text { least-squares } \\
\text { regressions with } \\
\text { the PROCESS tool } \\
\text { (88) }\end{array}$ & $\begin{array}{l}\text { Metacognitive beliefs about thoughts being } \\
\text { uncontrollable/dangerous significantly } \\
\text { mediated the relationship between early } \\
\text { emotional abuse and depression }\end{array}$ \\
\hline $\begin{array}{l}\text { Paredes and } \\
\text { Calvete (36); } \\
\text { Spain }\end{array}$ & Longitudinal & $\begin{array}{l}998 \text { adolescents ( } 471 \text { girls, } \\
526 \text { boys) between } 13 \text { and } \\
17 \text { years of age }\end{array}$ & $\begin{array}{l}\text { The 6-item version } \\
\text { of the Emotional } \\
\text { Abuse Scale } \\
\text { adapted from } \\
\text { CTS-PC }\end{array}$ & CRSS, ACSQ & CES-D & $\begin{array}{l}\text { Structural equation } \\
\text { modeling, Sobel } \\
\text { test }\end{array}$ & $\begin{array}{l}\text { Only brooding partially mediated the } \\
\text { relationship between emotional abuse by } \\
\text { parents and depressive symptoms. Neither } \\
\text { reflection nor negative inferential styles } \\
\text { increased vulnerability to depression }\end{array}$ \\
\hline $\begin{array}{l}\text { Raes and } \\
\text { Hermans (76); } \\
\text { Belgium }\end{array}$ & Cross-sectional & $\begin{array}{l}101 \text { students ( } 83 \text { women, } \\
\text { mean age }=19.64 \text { years) }\end{array}$ & $\begin{array}{l}\text { The emotional abuse } \\
\text { subscale of the CTQ }\end{array}$ & RRS & $\mathrm{BDI}$ & $\begin{array}{l}\text { Multiple regression } \\
\text { analyses, Sobel } \\
\text { test }\end{array}$ & $\begin{array}{l}\text { Brooding partially mediated the relationship } \\
\text { between emotional abuse and depression, } \\
\text { even when reflection was partialed out }\end{array}$ \\
\hline $\begin{array}{l}\text { Rafi et al. (77); } \\
\text { Iran }\end{array}$ & Cross-sectional & $\begin{array}{l}492 \text { middle school students } \\
(183 \text { boys, mean age }= \\
13.61 \text { years, } S D=0.682, \\
309 \text { girls mean age }=13.60, \\
S D=0.572)\end{array}$ & $\begin{array}{l}\text { Child Abuse Self } \\
\text { Report Scale }\end{array}$ & $\mathrm{SIC}$ & $\begin{array}{l}\text { The anxiety and } \\
\text { depression } \\
\text { subscales of the } \\
\text { ASEBA }\end{array}$ & Path analysis & $\begin{array}{l}\text { Early maladaptive schemas (i.e., loneliness, } \\
\text { vulnerability to harm, and submission) } \\
\text { mediated the relationship between childhood } \\
\text { emotional maltreatment and depression }\end{array}$ \\
\hline $\begin{array}{l}\text { Reddy et al. } \\
\text { (78); USA }\end{array}$ & Cross-sectional & $\begin{array}{l}987 \text { college undergraduates } \\
(52.5 \% \text { males, } 65 \% \\
\text { Caucasian, } 93 \% \text { below } 22 \\
\text { years old) }\end{array}$ & FEQ & $\begin{array}{l}\text { EAS, WBSI, } \\
\text { Acceptance and } \\
\text { Action Questionnaire }\end{array}$ & DASS-21 & $\begin{array}{l}\text { Structural } \\
\text { equation modeling }\end{array}$ & $\begin{array}{l}\text { Experiential avoidance (examined by three } \\
\text { measures) significantly mediated the } \\
\text { relationship between childhood psychological } \\
\text { abuse and current mental health symptoms } \\
\text { (including depression, anxiety and stress), } \\
\text { reducing the direct effect by } 77 \%\end{array}$ \\
\hline $\begin{array}{l}\text { Ross et al. } \\
\text { (53); USA }\end{array}$ & Cross-sectional & $\begin{array}{l}244 \text { adults }(53 \text { males; mean } \\
\text { age }=20.80, S D=3.826)\end{array}$ & CTQ & $\begin{array}{l}\text { ISS, the 26-item } \\
\text { Self-Compassion } \\
\text { Scale }\end{array}$ & CESD-R & $\begin{array}{l}\text { Path models, } \\
\text { bootstrapping }\end{array}$ & $\begin{array}{l}\text { A significant path from emotional abuse to } \\
\text { depression, and a significant indirect path that } \\
\text { passed through self-compassion and shame }\end{array}$ \\
\hline $\begin{array}{l}\text { Sachs- } \\
\text { Ericsson et al. } \\
\text { (79); USA }\end{array}$ & Cross-sectional & $\begin{array}{l}5614 \text { adults age range } \\
15-54 \text { years }(M=33.2 \\
\text { years, } S D=10.7)\end{array}$ & $\begin{array}{l}\text { A list of specific } \\
\text { behaviors related to } \\
\text { parental verbal } \\
\text { abuse, including } \\
\text { insulted, swore at, } \\
\text { did or said } \\
\text { something to spite, } \\
\text { threatened to hit. }\end{array}$ & $\begin{array}{l}4 \text { items of } \\
\text { self-criticism from } \\
\text { the DEQ, } \\
\text { Dependency and } \\
\text { Emotional Reliance } \\
\text { on Others Scale } \\
\text { (181) }\end{array}$ & CIDI & $\begin{array}{l}\text { Hierarchical linear } \\
\text { regression } \\
\text { analyses, Sobel } \\
\text { test }\end{array}$ & $\begin{array}{l}\text { Self-criticism fully mediated the relationship } \\
\text { between parental verbal abuse and } \\
\text { internalizing symptoms (including symptoms of } \\
\text { depression), whereas dependency did not } \\
\text { mediate the relationship between any abuse } \\
\text { and internalizing symptoms }\end{array}$ \\
\hline $\begin{array}{l}\text { Schulz et al. } \\
\text { (80); Germany }\end{array}$ & Longitudinal & $\begin{array}{l}123 \text { inpatients aged } 18-65 \\
\text { years with current MDD } \\
\text { diagnosis }\end{array}$ & $\begin{array}{l}\text { The emotional abuse } \\
\text { and emotional } \\
\text { neglect subscales of } \\
\text { the CTQ }\end{array}$ & PSDI, EAQ & $\begin{array}{l}\text { BDI-II, Montgomery- } \\
\text { Åsberg Depression } \\
\text { Rating Scale }\end{array}$ & (73), Sobel test & $\begin{array}{l}\text { Borderline personality traits and acceptance of } \\
\text { pleasant emotions are significant mediators of } \\
\text { the association between childhood emotional } \\
\text { abuse and self-rated depression severity. } \\
\text { Childhood emotional abuse is not correlated to } \\
\text { expert-ratings of depression }\end{array}$ \\
\hline
\end{tabular}

Structural

Structural equation modeling, Sobe parents and depressive symptoms. Neither reflection nor negative inferential styles creased vulnerability to depression even when reflection was partialed out mediated the relationship between childhood malional maltreatment and depression elationship between childhood psychological abuse and current mental health symptoms xiety and stress)

A significant path from emotional abuse to

Hierarchical linear egression analyses, Sobel and emotional

Åsberg Depression 


\begin{tabular}{|c|c|c|}
\hline $\begin{array}{l}\text { References; } \\
\text { Country }\end{array}$ & Design & Sample \\
\hline $\begin{array}{l}\text { Spasojevic } \\
\text { and Alloy ( } 81 \text { ); } \\
\text { USA }\end{array}$ & Longitudinal & $\begin{array}{l}137 \text { undergraduate students } \\
\text { aged } 16-29 \text { years ( } 88 \\
\text { females and } 49 \text { males, mean } \\
\text { age }=19 \text { ) }\end{array}$ \\
\hline $\begin{array}{l}\text { Uhrlass and } \\
\text { Gibb (82); } \\
\text { USA }\end{array}$ & Longitudinal & $\begin{array}{l}208 \text { undergraduate students } \\
\text { (mean age }=19.6 \text { years, SD } \\
=4.3,148 \text { females, } 58.2 \% \\
\text { Caucasian) }\end{array}$ \\
\hline $\begin{array}{l}\text { Van Assche } \\
\text { et al. (51); } \\
\text { Belgium }\end{array}$ & Cross-sectional & $\begin{array}{l}81 \text { older adults age range } \\
62-90(36 \% \text { males; } M= \\
74.90, S D=6.64)\end{array}$ \\
\hline
\end{tabular}

Measures

Childhood

emotional abuse

LEQ

LEQ

Q

LEQ

Mediats

RRS

BDI, Mod-SADS-L

The 53-item Hassles

subscale of the

Subscale of the
Hassles and Uplifts

Scale

CTQ-SF

ECR-R

GDS

BDI-II

Path analysis,
AMOS 5.0

Bootstrapping

van Harmelen
et al. (83); The

Cross-sectional

2837 adults aged between

18 and 65 years $(66.5 \%$

female, age $M=41.9$ years,

$S D=13.0)$

Wright et al.

Cross-sectional

301 undergraduate students

(143 men, 158 women

mean age $=20.37,94.4 \%$

Caucasian)

Yigit et al. Cross-sectional $\quad 325$ participants in total

(85); Turkey

clinical adolescents (129

girls; mean age $=15.65, S D$

$=1.15) ; 132$ non-clinical

adolescents (94 girls; mean

age $=15.05, S D=1.07)$

\section{$\begin{array}{ll}\text { The Netherlands } & \text { Implicit Association CIDI, IDS } \\ \text { Mental Health } & \text { Test }\end{array}$}

Survey and

Incidence Study

(NEMESIS) trauma

interview

LEQ

YSQ

TSC-40

TQ

Q

CTQ, Childhood Trauma Questionnaire; CTQ-SF, Childhood Trauma Questionnaire-Short Form; BDI, Beck Depression Inventory; BDI-II, Beck Depression Inventory II; YSQ, Young Schema Questionnaire; YSQ-SF, Young Schema Questionnaire-Short Form; YSQ-3, Young Schema Questionnaire-3; CECA, Childhood Experience of care and Abuse; CECA.Q, Childhood Experience of Care and Abuse questionnaire; HADS, Hospital Anxiety and Depression Scale; ECR-R, Experiences in Close Relationships Questionnaire-Revised; CATS, Child Abuse and Trauma Scale; SCID, Structured Clinical Interview for DSM-IV; SCID-I, Structured Clinical Interview for DSM-IV Axis 1 Disorders-Patient Edition; SCID-MDD, Structured Clinical Interview for DSM-III-R Non-Patient Version; DERS, Difficulties in Emotion Regulation Scale; ERQ, Emotion Regulation Questionnaire; IIP-32, Inventory of Interpersonal Problems; CSQ, Cognitive Style Questionnaire; DAS, Dysfunctional Attitude Scale; TSI, Trauma Symptom Inventory; BHS, Beck Hopelessness Scale; EDS, Emotion Dysregulation Scale; LEQ, Lifetime Experiences Questionnaire; NLEQ, Negative Life Events Questionnaire; LES, Life Experiences Survey; NEO-FFI, Neuroticism Extroversion Openness Five Factor Inventory; ECQ, Emotional Clarity Questionnaire; DASS-21, Depression Anxiety Stress Scale-21item; MASQ, Mood and Anxiety Symptom Questionnaire; WBSI, White Bear Thought Suppression Inventory; AEQ, Ambivalence Over Emotional Expressiveness Questionnaire; HVQ, History of Victimization Questionnaire; RFQ, Reflective Functioning Questionnaire; CDI, Children's Depression Inventory; CES-D, Center for Epidemiological Studies Depression Scale; CESD-R, Center for Epidemiological Studies Depression Scale-Revised; SADS, Schedule for Affective Disorders and Schizophrenia; Mod-SADS-L, A modified Schedule for Affective Disorders and Schizophrenia - Lifetime; HDSQ, Hopelessness Depression Symptom Questionnaire; ETI, Early Trauma Inventory; RRS, Ruminative Responses Scale; BADS, Behavioral Activation for Depression Scale; EPDS, Edinburgh Postnatal Depression Screen; SIC, A Schema Inventory for Children; ASEBA, Achenbach System of Empirically Based Assessment; FEQ, Family Experiences Questionnaire; ISS, Internalized Shame Scale; EAS, Experiential Avoidance Scale; PBRS, Personal Beliefs and Reactions Scale; GDS, Geriatric Depression Scale; EAQ, Emotion Acceptance Questionnaire; PSDI, Personality Style and Disorder Inventory; DEQ, Depressive Experiences Questionnaire; HRSD, Hamilton Rating Scale for Depression; ACSQ, Adolescent Cognitive Style Questionnaire; CRSS, Children's Response Style Scale; CTS-PC, Conflict Tactics Scales-Parent-to-Child;

TSC-40, Trauma Symptom Checklist-40; IDS, Inventory of Depressive Symptomatology; AAQ, Adult Attachment Questionnaire; CIDI, Composite International Diagnostic Interview. 
TABLE 2 | Quality assessment tool ratings.

\begin{tabular}{|c|c|c|c|c|c|c|c|c|}
\hline \multirow[t]{2}{*}{ References; Country } & \multirow{2}{*}{$\begin{array}{l}\text { Selection } \\
\text { bias }\end{array}$} & \multirow{2}{*}{$\begin{array}{l}\text { Study } \\
\text { design }\end{array}$} & \multirow[t]{2}{*}{ Confounders } & \multicolumn{3}{|c|}{ Data collection methods } & \multirow{2}{*}{$\begin{array}{l}\text { Withdrawals } \\
\text { and dropouts }\end{array}$} & \multirow{2}{*}{$\begin{array}{l}\text { Mediation } \\
\text { analyses }\end{array}$} \\
\hline & & & & IV & $\mathbf{M}$ & DV & & \\
\hline Calvete (62); Spain & W & M & W & S & S & S & M & S \\
\hline $\begin{array}{l}\text { Carvalho Fernando et al. (63); } \\
\text { Germany }\end{array}$ & M & W & $S$ & S & $S$ & $S$ & M & W \\
\hline Christ et al. (32); The Netherlands & W & W & $S$ & S & $\begin{array}{l}\text { Emotion dysregulation }=\mathrm{S} \\
\text { Interpersonal problems }=\mathrm{S}\end{array}$ & S & M & S \\
\hline $\begin{array}{l}\text { Coates and Messman-Moore (64); } \\
\text { USA }\end{array}$ & W & W & W & S & $\begin{array}{l}\text { Emotion dysregulation }=\mathrm{S} \\
\text { Negative internalized beliefs } \\
=\mathrm{S}\end{array}$ & $S$ & M & S \\
\hline Courtney et al. (65); USA & M & W & W & S & $S$ & $S$ & M & W \\
\hline Courtney et al. (30); USA & M & $\mathrm{M}$ & M & $S$ & $S$ & S & W & W \\
\hline Crow et al. (29); USA & $M$ & W & $S$ & $S$ & $\mathrm{M}$ & $S$ & $\mathrm{M}$ & $S$ \\
\hline Gibb et al. (66); USA & W & $M$ & $\mathrm{M}$ & $S$ & $S$ & $S$ & $S$ & W \\
\hline Gibb et al. (67); USA & W & W & W & $S$ & $S$ & $S$ & M & S \\
\hline $\begin{array}{ll}\text { Hankin (31); USA } & \text { Study } 1 \\
& \text { Study } 2\end{array}$ & $\begin{array}{l}\text { W } \\
\text { W }\end{array}$ & $\begin{array}{l}M \\
S\end{array}$ & $\begin{array}{l}S \\
S\end{array}$ & $\begin{array}{l}S \\
S\end{array}$ & $\begin{array}{l}\text { Insecure attachment style }= \\
\text { S } \\
\text { Negative cognitive style }=S \\
\text { Negative events }=S\end{array}$ & $\begin{array}{l}S \\
S\end{array}$ & $\begin{array}{l}S \\
S\end{array}$ & $\begin{array}{l}S \\
S\end{array}$ \\
\hline Hayashi et al. (69); Japan & M & W & W & S & $\begin{array}{l}\text { Personality }=\mathrm{S} \\
\text { Stress of life events }=\mathrm{S}\end{array}$ & S & M & S \\
\hline Jessar et al. (70); USA & W & M & M & S & $\mathrm{M}$ & $S$ & W & S \\
\hline Kaysen et al. (71); USA & M & W & W & W & $S$ & $S$ & M & W \\
\hline Khosravani et al. (52); Iran & $M$ & W & $S$ & S & S & $S$ & $\mathrm{M}$ & S \\
\hline Krause et al. (72); USA & W & W & W & W & $S$ & $S$ & $\mathrm{M}$ & $S$ \\
\hline Li et al. (54); UK & W & W & W & $S$ & $S$ & $S$ & M & $S$ \\
\hline Lumley and Harkness (28); Canada & M & W & M & S & S & $S$ & M & S \\
\hline Lumley and Harkness (89); Canada & W & W & $\mathrm{M}$ & $S$ & $S$ & $S$ & $\mathrm{M}$ & $\mathrm{M}$ \\
\hline Maciejewski and Mazure (74); USA & $\mathrm{M}$ & $\mathrm{M}$ & $S$ & S & $S$ & $S$ & M & W \\
\hline O'Mahen et al. (21); USA & M & W & M & S & $\begin{array}{l}\text { Brooding }=\mathrm{S} \\
\text { Behavioral avoidance }=\mathrm{S}\end{array}$ & $S$ & M & $S$ \\
\hline Østefjells et al. (75); Norway & M & W & $\mathrm{M}$ & $S$ & $S$ & $S$ & M & S \\
\hline Paredes and Calvete (36); Spain & W & $\mathrm{M}$ & W & S & $\begin{array}{l}\text { Brooding }=S \\
\text { Negative inferential styles } \\
=S\end{array}$ & S & $\mathrm{M}$ & S \\
\hline Raes and Hermans (76); Belgium & W & W & W & $S$ & $S$ & $S$ & M & M \\
\hline Rafi et al. (77); Iran & W & W & W & $S$ & W & W & $\mathrm{M}$ & S \\
\hline Reddy et al. (78); USA & W & W & $S$ & W & $S$ & S & $\mathrm{M}$ & S \\
\hline Ross et al. (53); USA & W & W & W & S & $\begin{array}{l}\text { Self-compassion }=\mathrm{S} \\
\text { Shame }=\mathrm{S}\end{array}$ & S & M & S \\
\hline Sachs-Ericsson et al. (79); USA & S & W & $S$ & W & $\begin{array}{l}\text { Self-criticism }=\mathrm{W} \\
\text { Dependency }=\mathrm{W}\end{array}$ & S & M & M \\
\hline Schulz et al. (80); Germany & M & M & $S$ & S & $\begin{array}{l}\text { Borderline personality }=\mathrm{S} \\
\text { Low acceptance of pleasant } \\
\text { emotions }=\mathrm{W}\end{array}$ & $S$ & W & S \\
\hline Spasojevic and Alloy (81); USA & W & M & M & S & S & $S$ & W & W \\
\hline Uhrlass and Gibb (82); USA & W & $\mathrm{M}$ & W & S & $S$ & $S$ & W & S \\
\hline Van Assche et al. (51); Belgium & $\mathrm{M}$ & W & $S$ & S & $S$ & $S$ & $\mathrm{M}$ & S \\
\hline $\begin{array}{l}\text { van Harmelen et al. (83); The } \\
\text { Netherlands }\end{array}$ & $S$ & W & $S$ & S & $S$ & $S$ & M & $S$ \\
\hline Wright et al. (84); USA & W & W & $S$ & $S$ & $S$ & S & M & W \\
\hline Yigit et al. (85); Turkey & $M$ & W & $\mathrm{M}$ & S & $S$ & S & M & $S$ \\
\hline
\end{tabular}

S, strong; $M$, moderate; $W$, weak; $N$, independent variable, $M$, mediator(s); DV, dependent variable. 
TABLE 3 | Summary of results and mediational analytic approaches.

\begin{tabular}{|c|c|c|c|c|c|}
\hline \multirow[t]{2}{*}{ Cluster } & \multirow[t]{2}{*}{ Eligible studies } & \multirow{2}{*}{$\begin{array}{l}\text { Percentage of studies } \\
\text { supporting the } \\
\text { pathway }\end{array}$} & \multicolumn{3}{|c|}{ Strength of the mediation analysis } \\
\hline & & & Strong & Moderate & Weak \\
\hline Early maladaptive schemas $(N=8)$ & $\begin{array}{l}\text { Calvete (62) } \\
\text { Coates and Messman-Moore (64) } \\
\text { Kaysen et al. (71) } \\
\text { Lumley and Harkness (28) } \\
\text { Lumley and Harkness (89) } \\
\text { Rafi et al. (77) } \\
\text { Wright et al. (84) } \\
\text { Yigit et al. (85) }\end{array}$ & $75 \%$ & $N=5$ & $N=1$ & $N=2$ \\
\hline Cognitive-personality variables $(N=14)$ & $\begin{array}{l}\text { Courtney et al. (65) } \\
\text { Courtney et al. (30) } \\
\text { Gibb et al. (66) } \\
\text { Gibb et al. (67) } \\
\text { Hankin (31) } \\
\text { Hayashi et al. (69) } \\
\text { Li et al. (54) } \\
\text { Maciejewski and Mazure (74) } \\
\text { Østefjells et al. (75) } \\
\text { Paredes and Calvete (36) } \\
\text { Ross et al. (53) } \\
\text { Sachs-Ericsson et al. (79) } \\
\text { Schulz et al. (80) } \\
\text { van Harmelen et al. (83) }\end{array}$ & $\begin{array}{l}92 \%(N=12 \text {; cognitive } \\
\text { vulnerability - the small } \\
\text { bandwidth of } \\
\text { cognitive-personality } \\
\text { variables) } \\
100 \% \text { ( } N=2 ; \\
\text { personality - the broader } \\
\text { cognitive- } \\
\text { personality variables) }\end{array}$ & $N=9$ & $N=1$ & $N=4$ \\
\hline Emotion dysregulation $(N=13)$ & $\begin{array}{l}\text { Carvalho Fernando et al. (63) } \\
\text { Christ et al. (32) } \\
\text { Coates and Messman-Moore (64) } \\
\text { Crow et al. (29) } \\
\text { Jessar et al. (70) } \\
\text { Khosravani et al. (52) } \\
\text { Krause et al. (72) } \\
\text { O'Mahen et al. (21) } \\
\text { Paredes and Calvete (36) } \\
\text { Raes and Hermans (76) } \\
\text { Reddy et al. (78) } \\
\text { Schulz et al. (80) } \\
\text { Spasojevic and Alloy (81) }\end{array}$ & $85 \%$ & $N=10$ & $N=1$ & $N=2$ \\
\hline Interpersonal styles $(N=4)$ & $\begin{array}{l}\text { Christ et al. (32) } \\
\text { Hankin (31) } \\
\text { Sachs-Ericsson et al. (79) } \\
\text { Van Assche et al. (51) }\end{array}$ & $50 \%$ & $N=3$ & $N=1$ & N/A \\
\hline Stressful negative events $(N=3)$ & $\begin{array}{l}\text { Hankin (31) } \\
\text { Hayashi et al. (69) } \\
\text { Uhrlass and Gibb (82) }\end{array}$ & $67 \%$ & $N=3$ & N/A & N/A \\
\hline
\end{tabular}

Nine studies examined more than one mediating variable in their analyses so might be counted more than once in different clusters in this table.

both men and women; four studies $(21,32,64,71)$ included female participants only and one study (52) included male participants only.

Sample sizes ranged from 50 to 5,614 participants. Twentynine studies had sample sizes larger than 100 . We used a sample size of 100 as guideline for performing mediation analyses, as MacKinnon et al. (90) argued that a sample size of 100-200 was sufficient even for multiple mediator models in terms of power. Nine studies examined more than one mediating variable in their analyses $(36,79)$. Of the 34 studies that tested mediation, seven were rated as weak, three as moderate, and 24 as strong. More than one-third $(n=13)$ studies lacked information or did not provide an interpretation for potential confounding variables, such as age, gender, and socioeconomic status. Eleven studies controlled for other types of childhood maltreatment (e.g., sexual and physical abuse) in the mediation analyses to disentangle the independent contribution of emotional abuse.

Among all eligible studies, six (17.6\%) found no association between childhood emotional abuse, mediating variables, and subsequent depression levels in their analyses. Van Assche et al. (51) reported that childhood emotional abuse was not significantly correlated with current depression or attachment 


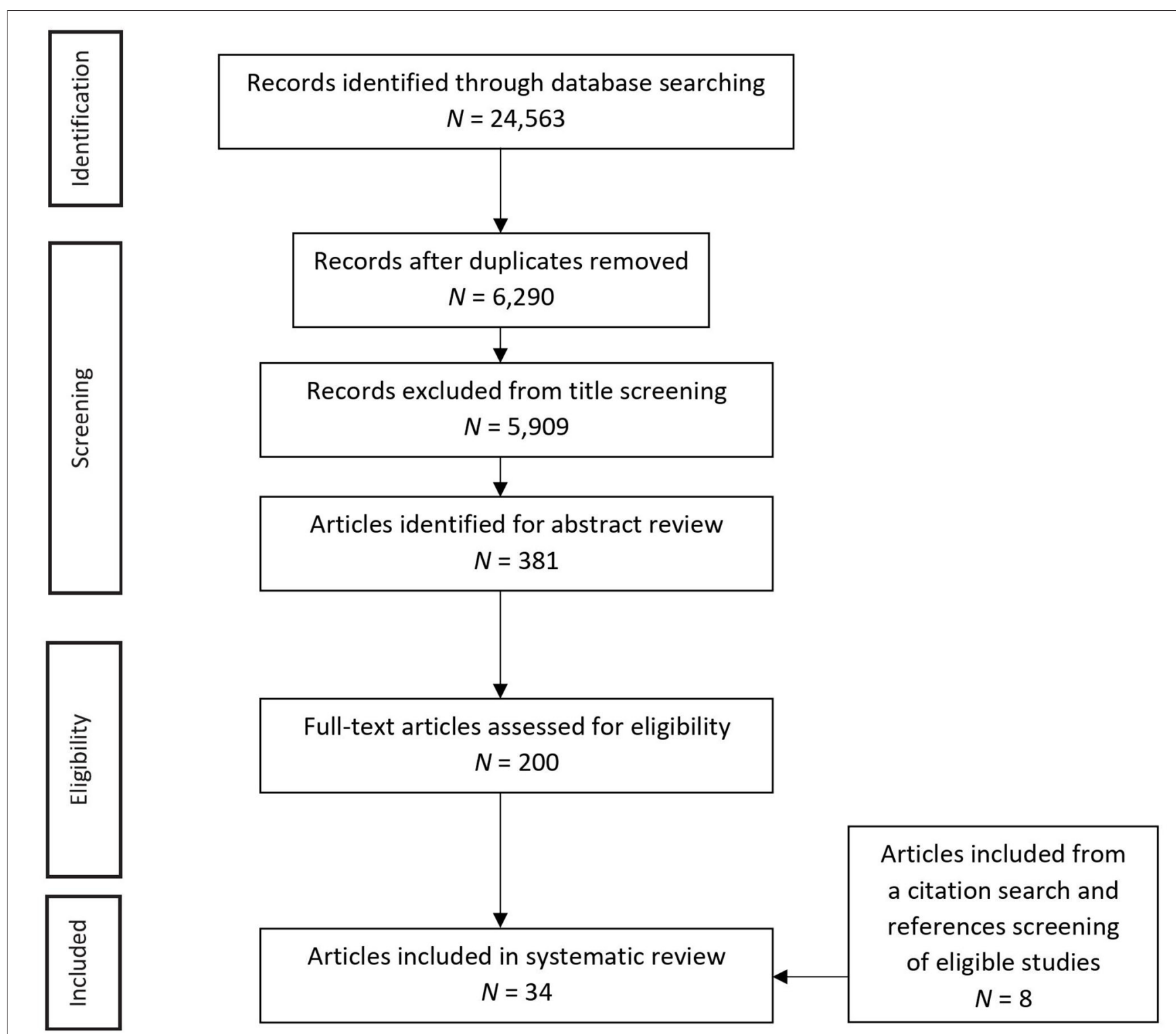

FIGURE 1 | Paper selection flow chart.

in a sample of community-dwelling older adults. Carvalho Fernando et al. (63) found no significant impact of selfreported childhood emotional abuse and emotion dysregulation on depressive symptoms. Hayashi et al. (69) reported that negative life events were affected by childhood abuse but did not predict the severity of depression. Kaysen et al. (71) showed no association of childhood emotional abuse with either adult depression or maladaptive cognitions. Calvete (62) reported that parental emotional abuse did not predict the worsening of early maladaptive schemas in adolescence, although it was directly associated with depressive symptoms. Jessar et al. (70) also indicated that emotional abuse did not significantly predict deficits in emotional clarity but did predict increases in depressive symptoms.
In addition, no specificity was found in the relation between emotional abuse and anhedonic depression in Lumley and Harkness's (28) analysis. Unexpectedly, Schulz et al. (80) reported that childhood emotional abuse was not correlated with expert ratings of depression, although it was correlated with self-rated depression severity. Some of these findings might be caused by the considerable co-occurrence of childhood sexual, physical, and emotional abuse in the studied samples. Any independent effects of emotional abuse on the development of depression might be diminished when severe physical or sexual abuse is also present. Therefore, we suggest that it would be important for future research to assess exposure to all forms of childhood maltreatment simultaneously, as multiple types of maltreatment may interact to produce varying outcomes, and to rule out other 
forms of maltreatment that may have been contributing factors when examining the emotional abuse-depression link.

\section{Overview of the Measures Used in the Primary Studies \\ Childhood Emotional Abuse}

Following methodological recommendations from PRISMA (49), a component approach to quality assessment was employed. Regarding the measures used in the eligible studies, reliability and validity were taken into consideration. According to the EPHPP, (a) standard assessment tools with known reliability and validity were rated as strong; (b) data collection tools that have been shown to be valid but not shown to be reliable (or for which reliability is not described) were rated as moderate; and (c) measures that have not been shown to be valid (or for which both reliability and validity are not described) were rated as weak. Four studies obtained weak ratings for measures employed to capture childhood emotional abuse. The remaining studies employed valid and reliable measures of the independent variables being investigated. The most widely used retrospective measures of childhood emotional abuse were the CTQ $(91,92)(n=14)$ and the LEQ (93) $(n=6)$. Research has provided considerable support for the reliability and validity of both scales. The CTQ is a 28-item self-report inventory developed to measure five types of abuse or neglect in childhood or adolescence. Five CTQ items directly assess verbal and non-verbal emotional abuse: (1) People in my family called me things like "stupid," "lazy," or "ugly"; (2) I thought that my parents wished I had never been born; (3) People in my family said hurtful or insulting things to me; (4) I felt that someone in my family hated me; (5) I believe that I was emotionally abused. Participants rate each item on a 5-point Likert-type scale with higher scores indicating more emotional abuse. The CTQ also provides cut-off scores for none to low, low to moderate, moderate to severe, and severe to extreme trauma exposure, and the cut-off scores for moderate to severe maltreatment are 13 or higher for emotional abuse (92). The LEQ scenarios describe specific examples of emotional, physical, and sexual abuse. Participants are instructed to indicate whether these events had occurred, the age of onset for each maltreatment event, cessation of the event, the frequency of occurrence, and the perpetrator, rather than reporting on global estimates of abuse. Twenty-seven items in the LEQ assess emotional abuse, including forms of belittling, ridiculing, spurning, humiliating, rejecting, extorting, and terrorizing. For example, "Did anyone humiliate or demean you in the presence of other people?" Only three studies adopted interview measures. Two studies $(28,31)$ used the CECA (94). The CECA is a semi-structured contextual threat interview that assesses childhood adversity, including the familial context of quality of care and relationship with parents or substitutes (i.e., antipathy, emotional neglect, discipline, discord) in addition to psychological, physical, and sexual abuse.

\section{Depression}

The operationalization of depression varied greatly. Using EPHPP criteria, one study (77) obtained a weak rating for the measure employed to capture depression symptoms; the remaining studies employed valid and reliable measures of the dependent variables being investigated. Most studies examined symptoms of depression as the outcome variable in community samples. Among the 34 eligible studies, only seven were conducted with clinical samples $(28,63,69,74,75,80,85)$. Seventeen studies used the BDI and the BDI-II $(95,96)$, four used the CES-D (97), two used the DASS-21 (98), two used the MASQ (99), and two used the CDI (100).

Nine studies $(21,28,66,71,74,79,81,83,89)$ used interview measures to assess the severity of depression or diagnosis. Four studies $(21,71,74,89)$ were conducted with different versions of the SCID (101) to assess major depressive disorder (MDD) as defined in DSM-IV (102). Three studies $(74,79,83)$ used the CIDI (103) also based on DSM-IV criteria for MDD (102). Three studies $(28,66,81)$ used different versions of the SADS (104) to assess episodes of depression.

\section{Mediators}

A range of potential mediators was assessed by various measures. According to the EPHPP, five studies obtained weak or moderate ratings for measures employed to capture the mediating variable; the remaining studies employed valid and reliable measures of the mediators being investigated.

\section{Synthesis of the Mediators of the Emotional Abuse-Depression Relationship}

For the purposes of the review, we grouped studies according to the type of psychological mechanisms examined in the emotional abuse-depression link. These mediators were further grouped according to the five clusters of psychological mediators: early maladaptive schemas, cognitive-personality variables, emotion dysregulation, interpersonal styles, and stressful negative events (see Figure 2). These domains were created as part of the narrative integration approach we used to organize the findings from the literature. Eight studies had analytic approaches rated as weak (e.g., regression methods) on their appropriateness in terms of testing for mediation effects, three studies were rated as moderate (e.g., regression methods with additional Sobel test), and the remaining 23 studies were rated as strong (e.g., bootstrapping techniques; path analysis). Table 3 provides a summary of results and mediational analytic approaches.

First, a large body of cross-sectional and prospective research has highlighted the role of negative schema contents in depression. Early maladaptive schemas (105) are defined as broad, dysfunctional, and pervasive patterns consisting of memories, emotions, cognitions, and bodily sensations about oneself and relationships with others and hypothesized to consolidate over time. Certain schema domains (disconnection/rejection, impaired autonomy and performance, and other directedness) have been found to be associated with depressive symptoms (106).

Second, theories that focus on personality dimensions include broad-bandwidth theories focusing on the Big Five dimensions (107), and narrower bandwidth theories focusing on more specific personality dimensions, such as sociotropy-autonomy $(108,109)$ and cognitive vulnerability as conceptualized in Beck's original formulation of the cognitive model of depression $(108,110)$. In this review, we grouped cognitive vulnerability 


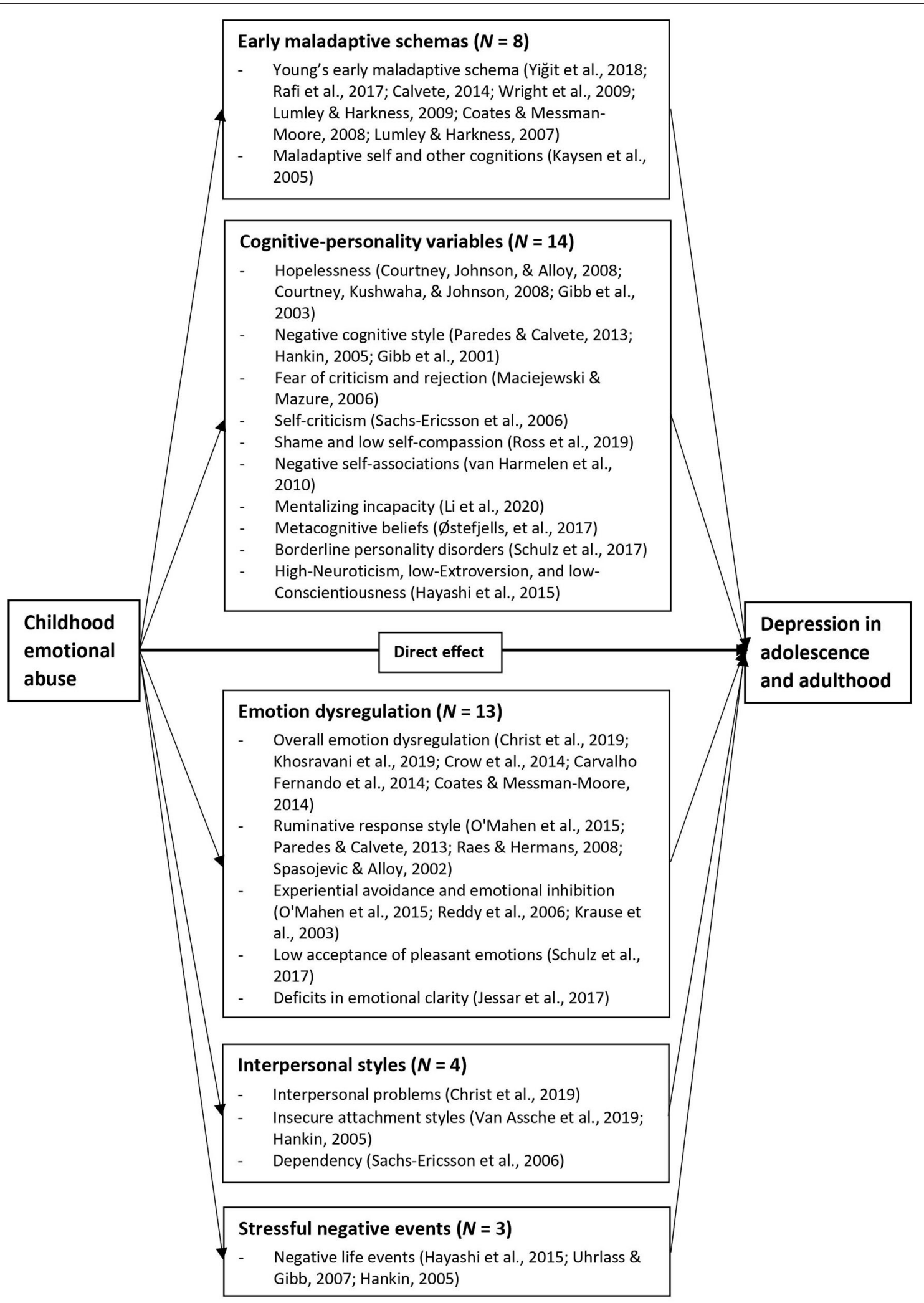

FIGURE 2 | Examined mediators in the emotional abuse-depression link. 
with personality traits and dysfunctions as cognitive-personality variables. Cognitive theories of depression (111) have postulated that the impact of childhood maltreatment on subsequent psychopathology might be mediated by cognitive vulnerability (i.e., relatively stable, trait-like negative cognitive styles). People with chronic experiences of childhood emotional abuse, as opposed to physical or sexual abuse, are more likely to interpret current negative events in a similarly depressogenic manner (e.g., negative self-associations) because the depressogenic interpretations are directly supplied by the abusers (e.g., "you are such a stupid child"), which accounts for subsequent increases in depressive symptoms (61). A large number of studies have shown dysfunctional attributional styles and negative inferential styles for causes, consequences, and self-characteristics as predictors of depression in clinical and non-clinical samples (112-114). In addition, high Neuroticism, low Extroversion, and low Conscientiousness in the five-factor model of personality have been consistently linked to the onset of MDD (115). Although traits are considered to be largely genetically determined, neuroticism has been found to change over time as a result of childhood maltreatment (116). Several studies also revealed associations between childhood emotional abuse and different areas of personality dysfunction, including borderline, avoidant, and dependent pathology $(117,118)$. Given the high prevalence of comorbid personality disorders in inpatients with MDD (119), changes in personality functioning may mediate the emotional abuse-depression link. In Beck's (108) models of depression, these cognitive-personality characteristics have been shown not to be simply latent diatheses for depression; instead, they increase the probability of particular life events and one's vulnerability for the onset of depression with a diathesis-stress nature (120).

Third, chronic experiences of parental emotional abuse may increase children's internal focus and lead to emotion dysregulation, such as rumination. Individuals who tend to ruminate in response to negative life events are at high risk of developing and maintaining depressive symptoms. Treynor et al. (121) identified two distinct rumination components, labeled as brooding and reflection. There is a body of research consistently linking brooding rumination, but not reflection, with the onset and maintenance of depression (122).

Fourth, by definition, emotional abuse encompasses a repeated pattern of maladaptive interactions between the parent and child and has been described as a "pathogenic relational environment" [(123), p. 414]. Exposure to emotional abuse clearly threatens the security of attachment relationships and results in maladaptive models of self and self-in-relationto-others, which may then impact on the quality of later interpersonal relationships. Individuals with a history of childhood maltreatment in general reported more dysfunctional relationships (124) and lower levels of social support (34) in adulthood. Recurring frustrated interpersonal dynamics, in turn, will render an individual more vulnerable to depression (125). Although empirical research is still scarce, interpersonal problems have been thought to be an underlying factor of the emotional abuse-depression link $(126,127)$.

Finally, Hammen's $(128,129)$ stress generation model of depression posited that a reciprocal relationship exists between negative life events and depression, whereby depressed individuals may generate additional negative stressors as they seek reassurance and comfort from others, only to be rejected by those they turned to for social support, and thus actively contribute to the occurrence of negative events in their lives. Importantly, Hammen's model suggests that childhood emotional abuse may set the stage for self-generated negative events and depressive symptoms in adolescence and adulthood (130).

\section{Early Maladaptive Schemas}

In total, eight studies examined early maladaptive schemas as the pathway by which childhood emotional abuse may lead to subsequent depression symptoms. Both cross-sectional $(28,64$, $71,77,84,85,89)$ and longitudinal (62) designs were employed. Five $(28,62,64,84,89)$ used the YSQ $(105)$ and two $(77,85)$ adapted the YSQ for Turkish or Iranian adolescents.

Four studies examined particular schema themes that may mediate the emotional abuse-depression link $(28,77,84,85)$. Lumley and Harkness (28) found that specific early maladaptive schemas of social isolation and self-sacrifice mediated the relation between early emotional maltreatment and anhedonic depression in depressed adolescents. Wright et al. (84) found that the schemas of vulnerability to harm, self-sacrifice, and defectiveness/shame mediated the relationship between childhood emotional abuse and symptoms of depression in a general population of young adults. In Turkish adolescents, Yigit et al. (85) found that both disconnection/rejection and impaired autonomy significantly mediated emotional abuse and depression in their non-clinical sample, whereas only disconnection/rejection significantly mediated the relationship between emotional abuse and depression in their clinical sample. In Iranian adolescents, Rafi et al. (77) reported that early maladaptive schemas of loneliness, vulnerability to harm, and submission mediated the relationship between childhood emotional maltreatment and depression. However, neither the Child Abuse Self Report Scale (CASRS) for assessing childhood emotional maltreatment nor the Schema Inventory for Children (SIC) for measuring early maladaptive schemas used in the study of Rafi et al. was validated. The CASRS was created in a master's thesis (131) and not peer-reviewed or published, and no further evidence of validity or reliability was found for the SIC.

In another two studies using the YSQ, Coates and MessmanMoore (64) found that general negative internalized beliefs significantly mediated the link between childhood psychological maltreatment and depressive symptoms. Lumley and Harkness (89) used a computer task, the Modified-Psychological Distance Scaling Task (M-PDST), whose stimuli (i.e., schema statements) were derived from the YSQ, to examine depressotypic schema organization as a mediator in young adults. They found that tightly connected negative schema statements mediated the relation between maternal and paternal emotional maltreatment and current depression, whereas loosely connected positive schema statements mediated the relation between maternal emotional maltreatment and current depression.

Unexpectedly, two studies $(62,71)$ found no association of parental emotional abuse with depression and/or maladaptive 
schemas. Calvete (62) reported that parental emotional abuse predicted adolescents' depressive symptoms but not a worsening of early maladaptive schemas over time. Regardless, parental emotional abuse might have influenced the origin of early maladaptive schemas before their study. Kaysen et al. (71) found no association of childhood emotional abuse with depression or maladaptive cognitions in a sample of adult women who had recently been raped or physically assaulted. However, they used only three items (i.e., being called bad, dumb, or stupid; being threatened with a beating; being cursed at) of the HVQ to measure experiences of childhood emotional abuse in a recently traumatized population, and did not rule out other forms of maltreatment that are very likely to be contributing factors. This limits the individual effects of childhood emotional abuse on subsequent maladaptive cognitions and depressive symptoms.

Overall, six out of eight (75\%) studies $(28,64,77,84,85,89)$ supported early maladaptive schemas as the pathway by which childhood emotional abuse may lead to subsequent depression symptoms. However, there is a lack of consistency in their findings concerning whether maladaptive schemas in general or specific schema themes act as the mediator. Only the schemas of vulnerability to harm and self-sacrifice appeared as the mediators in the emotional abuse-depression link more than once. Overall, two studies were rated as weak $(71,84)$, one as moderate $(89)$, and five as strong, in terms of the relative strength of the statistical mediation analysis.

\section{Cognitive-Personality Variables}

In total, 14 studies examined cognitive-personality variables as the pathway by which childhood emotional abuse may lead to subsequent depression symptoms. Within this domain, we split cognitive-personality variables into two subgroups (narrow bandwidth vs. broad bandwidth): one collection of studies ( $n=$ 12) has drawn on cognitive processes focusing on specific types of cognitive vulnerability, whereas another tradition has focused on broader cognitive-personality factors $(n=2)$.

In the subgroup of studies focusing on cognitive vulnerability, three studies $(30,65,67)$ reported hopelessness as a mediator in the emotional abuse-depression link in adolescent or young adult samples using the BHS. Courtney et al. (65) found that the association of emotional abuse with depressive symptoms remained significant even after controlling for hopelessness, indicating that hopelessness was a partial mediator of the association between emotional abuse and depressive symptoms. In line with them, Gibb et al. (67) also indicated that the etiological chain of the hopelessness theory mediates only part of the relation between emotional maltreatment and symptoms of hopelessness depression. However, Courtney et al. (30) showed that hopelessness accounted for $87.3 \%$ of the variance in this association where the association of emotional abuse index scores with depression did not remain significant after controlling for hopelessness. Nonetheless, Courtney et al. (30) used only three items adapted from the CTQ that directly assess verbal and nonverbal emotional abuse and did not rule out other forms of maltreatment that may have been contributing factors, which limits the individual effects of emotional abuse on subsequent hopelessness and depressive symptoms.
Three studies $(31,36,66)$ examined cognitive vulnerability as a potential mediating variable using the CSQ. Gibb et al. (66) found that cognitive risk fully mediated the relation of childhood emotional abuse to non-endogenous major depression and hopelessness depression, respectively, in a 2.5 -years followup study. Hankin (31) also found that cognitive vulnerability helped account for the emotional abuse-depression link in two separate studies, a 10-weeks and a 2-years longitudinal study with undergraduate students. However, Paredes and Calvete (36) showed no evidence for negative inferential styles to be a mediator in their follow-up study. They reported that only negative inferential styles for consequences predicted depressive symptoms, which notably contrasts with studies showing that inferential style for causes of negative events is the most important element in the prediction of depressive symptoms $(112,113,132)$. The CSQ employed in the above three studies is a self-report instrument assessing explicit cognitions. To include both explicit and automatic processes of cognitive functioning, van Harmelen et al. (83) had 2,837 adult participants complete a computer task, the Implicit Association Test (IAT). They found that childhood emotional maltreatment had the strongest link with enhanced explicit and automatic self-depression associations, compared with physical and sexual abuse; moreover, an increase in explicit and automatic negative self-associations partially mediated the relation between childhood emotional maltreatment and depressive symptomatology.

Three studies $(53,74,79)$ assessed specific negative cognitive styles as mediators in the emotional abuse-depression link: fear of criticism and rejection (74), self-criticism (79), and shame and low self-compassion (53). Self-criticism, self-blame, and noncompassionate introjects may underlie the feelings of shame with a harsh internalized voice. All of them were found to significantly mediate the emotional abuse-depression link in adult samples. A significant path from emotional abuse to depression and a significant indirect path that passed through self-compassion and shame were reported by Ross et al. (53). However, after adjusting for fear of criticism and rejection, perceived childhood emotional abuse was no longer significantly associated with major depression in another study (74). Although Sachs-Ericsson et al. (79) also reported that verbal abuse no longer predicted depression once self-criticism was included in the analyses, they used specific behaviors of verbal abuse (e.g., insulted, swore at, did or said something to spite, threatened to hit) to estimate emotional abuse, which was neither validated nor shown to be reliable.

Finally, two studies $(54,75)$ examined metacognition as a mediator in the emotional abuse-depression link. Li et al. (54) found that childhood emotional abuse continued to exert a significant effect on adulthood depression after controlling for other forms of early maltreatment and current mentalizing incapacity in a general sample of 205 adults. A mediation effect between childhood emotional abuse and adulthood depression symptoms via current mentalizing incapacity (both hypermentalizing and hypomentalizing) was established in their analysis. Østefjells et al. (75) reported that specific beliefs about thoughts being uncontrollable and dangerous significantly mediated the relationship between early emotional abuse and 
depression in 261 adult patients with psychotic or bipolar disorders. Although both studies examined metacognitionrelated mediators, Li et al. (54) introduced mentalizing to capture the interpersonal, cognitive, and developmental constructs of depression as an umbrella term for a group of basic psychological processes (e.g., theory of mind, reflective functioning), whereas metacognitive beliefs in Østefjells et al. (75) cover only specific beliefs about thoughts being uncontrollable and dangerous.

In the subgroup with broad bandwidth, two studies examined personality as a mediator in clinical adult samples by employing a cross-sectional (69) and a prospective observational (80) design. Hayashi et al. (69) found that childhood emotional abuse predicted the severity of depression indirectly through the mediation of high Neuroticism, low Extroversion, and low Conscientiousness in Japanese MDD patients. Schulz et al. (80) reported that elevated borderline personality traits mediated the association of childhood emotional abuse and selfrated MDD symptoms in German MDD patients; additionally, elevated passive-aggressive personality disorder traits mediated the link between childhood emotional abuse and lower selfrated symptom improvement. Although both studies examined personality as a potential mediator, Hayashi et al. (69) measured the five personality domains described in the five-factor model (107) using the NEO-FFI, whereas Schulz et al. (80) assessed personality traits using the PSDI. Unlike the NEO-FFI, the PSDI allows assessment of DSM-IV and ICD-10 personality traits as a dimensional approach in which characteristics of specific personality traits vary from adaptive to clinical levels and further reflect a personality disorder. Hayashi et al. (69) conducted structural equation modeling to examine the mediation model, and Schulz et al. (80) estimated direct and indirect effects using Preacher and Hayes (73) and the Sobel test; both were therefore rated as strong.

Overall, 11 of 12 (92\%) studies drawing on cognitive processes established cognitive vulnerability, including hopelessness, general and specific types of negative cognitive styles, and metacognition, as mediators in the emotional abuse-depression link. Two studies assessing personality as a mediator potentially supported broader cognitive-personality factors as the pathway. There appears to be consistency of evidence supporting cognitive vulnerability (the small bandwidth of cognitive-personality variables; $n=11$ ) as the pathway based on a noticeably greater number of studies, in contrast to personality (the broader cognitive-personality variables; $n=2$ ). Although the above outcomes suggest that cognitive vulnerability may represent a common mediating mechanism in the emotional abusedepression link, four were rated as weak $(30,65,66,74)$ and one as moderate (79) in terms of the analytic approach employed to test for mediation effects. Hence, further research is needed to replicate the abovementioned outcomes with explicit analyses estimating direct and indirect effects (e.g., bootstrapping techniques).

\section{Emotion Dysregulation}

In total, 13 studies examined emotion dysregulation as a potential mediating variable in the emotional abuse-depression link. Overall emotion dysregulation was examined in five studies
(29, 32, 52, 63, 64). Two studies $(32,64)$ reported that the effect of childhood emotional abuse on depressive symptoms was significantly mediated by emotion dysregulation measured by the DERS in emerging adult women. Khosravani et al. (52) reported a direct effect of emotional abuse on depressive symptoms and an indirect effect via emotion dysregulation, also measured by the DERS, in a sample of 350 treatmentseeking males with heroin dependence. Consistently, Crow et al. (29) found that emotion dysregulation partially mediated the relationship between childhood emotional abuse and later depression in a low-income African American sample of 3,902 adults, using the EDS. However, no significant impact of selfreported childhood emotional abuse and emotion dysregulation on depressive symptoms was found in a case-control sample by Carvalho Fernando et al. (63), although they reported that a history of emotional abuse was uniquely related to more frequent use of expressive suppression.

Eight studies examined specific dysfunctional emotion regulation strategies (e.g., rumination, emotional inhibition, experiential avoidance, low acceptance of pleasant emotions) as potential mediating variables. These dysfunctional emotional regulation strategies are largely overlapping and mutually influencing constructs. Reddy et al. (78) found that increased levels of experiential avoidance significantly mediated the relationship between childhood psychological abuse and current symptoms of depression in a cross-sectional sample of 987 undergraduates, reducing the direct effect by $77 \%$. Experiential avoidance contains the elements of emotional inhibition and behavioral avoidance. In support of Reddy et al. (78), Krause et al. (72) found that chronic emotional inhibition fully mediated the relationship between a history of parental psychological abuse and adult depressive symptoms in a cross-sectional study. While employing an experientially avoidant coping style to inhibit thoughts and feelings may be temporarily adaptive as a means of escaping aversive emotional experiences, it often paradoxically leads to increased rumination on the thing sought to be avoided (78).

A ruminative response style was specifically examined as a potential mediating variable in four studies $(21,36,76,81)$. Spasojević and Alloy (81) first examined and found that rumination partially mediated the relationship between childhood emotional maltreatment and major depressive episodes in young adults who were followed prospectively for 2.5 years. The other three studies $(21,36,76)$ all reported that brooding rumination (not reflection) partially mediated the relationship between emotional abuse and depressive symptoms in samples of adolescents, young adults, and low-income pregnant women. They suggested that the mediating role of rumination would be attributable to the brooding form, rather than to the more adaptive component of reflection. As the relationship between brooding and behavioral avoidance was hypothesized to be reciprocal (133), O'Mahen et al. (21) also examined behavioral avoidance in their study but found no correlation between childhood emotional abuse and behavioral avoidance.

Moreover, Schulz et al. (80) reported that low acceptance of pleasant emotions mediated the association between childhood 
emotional abuse and self-rated depressive symptoms in a longitudinal study of 123 German MDD inpatients. Finally, Jessar et al. (70) assessed emotional clarity as a mediator between childhood emotional maltreatment and adolescent depression in a longitudinal study, but found that emotional abuse did not significantly predict deficits in emotional clarity.

In total, 11 of $13(85 \%)$ studies in this cluster supported emotion dysregulation as a mediator in the emotional abusedepression link. Overall emotion dysregulation $(n=4)$ and brooding rumination $(n=4)$ appeared to be the most consistent mediators in this link. In terms of the analytic approach employed to test for mediation effects, all studies in this cluster scored strong, except for two studies $(63,81)$ rated as weak, and one study (76) rated as moderate.

\section{Interpersonal Styles}

Four studies $(31,32,51,79)$ examined interpersonal styles as the pathway by which childhood emotional abuse may lead to subsequent depression symptoms. Hankin (31) reported that a more insecure attachment style prospectively (over both 10 -weeks and 2-years follow-up) mediated the association between a childhood history of emotional maltreatment and depressive symptoms in young adults. However, in a sample of older adults aged 62 and older, Van Assche et al. (51) found no significant correlation of childhood emotional abuse with either insecure attachment or current level of depression. Christ et al. (32) found that the effect of childhood emotional abuse on depressive symptoms was significantly mediated by two domains of interpersonal problems: cold/distant and domineering/controlling. Finally, Sachs-Ericsson et al. (79) unexpectedly found that dependent characteristics did not mediate the relationship between any parental abuse and depression symptoms in a large sample of adults, although a dependent interpersonal style has been constantly posited as a vulnerability marker for depression in literature. Three studies scored strong in their mediational analytic approaches (31, 32, 51) and one (79) was rated as moderate. Overall, only half of the studies in this cluster $(31,32)$ found expected outcomes supporting insecure attachment styles and specific interpersonal styles as potential pathways in the emotional abuse-depression link.

\section{Stressful Negative Events}

Three studies examined stressful negative events as a potential mediating variable in the emotional abuse-depression link (31, $69,82)$. Two studies $(31,82)$ employed a longitudinal design with path analysis in young adults. Uhrlass and Gibb (82) found that changes in recent negative events fully mediated, rather than moderated, the link between childhood emotional maltreatment and depressive symptoms; additionally, initial depressive symptoms contributed to prospective changes in negative life events. Instead of assessing major life events (e.g., deaths or unemployment), the Hassles Scale used in Uhrlass and Gibb's 7-weeks longitudinal study calculated the level of negative life events by summing the number of hassles endorsed during the past week. Hankin (31), described above, also assessed negative life events as a potential mediator using NLEQ which examines negative life events that typically occur for young adults. They found that experiencing a greater number of negative life events prospectively (over both 10-weeks and 2years follow-up) mediated the association between a childhood history of emotional maltreatment and depressive symptoms in young adulthood. Unexpectedly, Hayashi et al. (69) found that negative life change affected by childhood emotional abuse did not predict the severity of depression. Although these three studies all scored strong in their mediational analytic approaches, only two of them supported stressful negative events as a potential mediating variable.

\section{DISCUSSION}

Existing reviews $(24,33,39,40)$ have focused on the psychological mechanisms leading from childhood maltreatment in general to adult depressive symptoms, without considering the specific contributions of subtypes of maltreatment. The current systematic review goes beyond previous findings by examining potential mediating variables specifically in the emotional abuse-depression link. The aims of this review were to provide a comprehensive systematic review of quantitative literature investigating potential psychological mediators of the association between childhood emotional abuse and dimensional and categorical depression symptoms, with a focus on the quality of this evidence, including the relative strength of the statistical mediation analyses used to explain the emotional abusedepression link. Our findings demonstrate several potentially causal mechanisms that might be involved in the relation between childhood emotional abuse and adolescent and adult depression. These psychological mediators fall into five clusters: (1) early maladaptive schemas, (2) cognitive-personality variables, (3) emotion dysregulation, (4) interpersonal styles, and (5) stressful negative events.

An important limitation of the eligible studies lies in the fact that not all of them addressed the unique impact of emotional abuse by accounting for the effects of the other types of childhood maltreatment. Only one-third of studies $(n=11)$ identified the unique contribution of emotional abuse to depression by statistically controlling for a history of other forms of childhood maltreatment $(29,31,32,53,54,63,78-80,83,84)$. Emotional abuse was found to be uniquely associated with later depression after partialing out the effect of other forms of childhood maltreatment, and the findings of these 11 studies remained essentially unchanged when covariates were removed. Wright et al. (84) reported that the intercorrelations between all the abuse variables ranged from a low of 0.16 (between sexual abuse and emotional neglect) to a high of 0.63 (between physical abuse and emotional abuse), suggesting that different forms of child maltreatment do covary with each other and often co-occur in the same household. Given the co-occurring forms of childhood abuse, neglect, and other adverse family experiences, it would be of importance for future mediational studies to model the concurrent and possible additive effects of different types of abuse. In addition, a few studies (72) did not report the strength of the direct effect between the emotional abuse latent variable 
and the depression latent variable without the mediator latent variable in the model. As such, a level of caution is required in interpreting the relative contributions of emotional abuse and mediators to ongoing depression.

Most eligible studies focused on examining a single mediator and indicated that this particular factor had the most impact in leading to depression following childhood emotional abuse. Nine studies included more than one variable in the mediational model of the emotional abuse-depression link (21, 31, 32, 36, 53, 64, 69, 79, 80), mainly involving emotion dysregulation, negative cognitive styles, stressful negative events, interpersonal styles, and personality, suggesting that the pathway from emotional abuse to depression is multi-determined and involves both cognitive and emotion-related factors. However, when integrating more than one mediator in a predictive model, not only effects and causal inferences might overlap with each other, but bidirectional relationships might also exist among contributing variables. For example, studies on stress generation $(106,134)$ have suggested that temperament may be related to stress-generation and increase interpersonal difficulties. Moreover, the cognitive and emotional deficits in the development of depression are not necessarily exclusive; studies have suggested an important role of cognitive processes underlying the regulation of emotion (135). Coates and Messman-Moore (64) reported that negative internalized beliefs increased vulnerability to emotion dysregulation and showed that the latter was an eventual outcome in mediating the emotional abuse-depression link.

Furthermore, as some mediating variables may be significant only when tested in isolation and not when entered simultaneously with other mediating variables, controlling for the interrelation between psychological mediators is important. Hankin (31), for instance, reported that an insecure attachment style and negative life events almost completely mediated the association between childhood emotional abuse and later depressive symptoms, while a negative cognitive style was minimized in their multivariate mediational model. Similarly, Coates and Messman-Moore (64) found that negative internalized beliefs no longer predicted depressive symptoms where emotion dysregulation appeared to be the stronger factor when the two were simultaneously entered into the same model. Likewise, a ruminative response style consistently appeared to be a stronger factor when entered simultaneously with cognitive vulnerability competing in the same mediational model $(36,81)$. Thus, our review supports the notion that not one mediator in isolation, but complex interrelations of psychological mediators, affect the relationship between childhood emotional abuse and subsequent depression.

Finally, although this review has shown specific pathways from exposure to childhood emotional abuse to later depression, the effects of childhood adversities are considered multivariate in their ultimate presentation (136). It has been suggested that childhood emotional abuse could first lead to the onset of depression in children and adolescents, and that depression in childhood and adolescence confer risk for depression in adulthood (137). A distal history of childhood maltreatment and context does not invariably contribute to depressive symptoms in adolescence and adulthood. Hamilton et al. (138) found that emotional abuse occurring during adolescence contributed to depressive symptoms in this period. Liu et al. (15) found that ongoing emotional maltreatment predicted shorter time to first onset of MDD in a sample of young adults. These findings together suggest that although childhood emotional abuse may have a specific depressogenic effect over a longer period of time, emotional abuse that occurs during that developmental stage may contribute to depression in a more immediate period. Further research is required to determine the specific contextual and process-related factors underlying this relationship.

\section{Toward a More Comprehensive Theoretical Model}

In most eligible studies reporting mediations, childhood emotional abuse still significantly predicted depression symptoms after accounting for potential mediators, suggesting that each examined mediator is only one of many potential pathways in the emotional abuse-depression link. However, the psychological mediators examined in the emotional abusedepression link in previous studies are largely dependent on researchers' preferences. Moreover, there are big differences in the conceptual and epistemological status of the various mediators that have been investigated. Person-dependent stressful life events, for instance, focus on the interplay between the individual and his/her environment, while rumination focuses on intrapsychic processes. Yet, it must be clear that rumination may also contribute to stress-generation processes, as do higher order theoretical constructs, such as personality. Hence, the current review suggests that there is a great degree of overlap in the investigated mediators between childhood emotional abuse and depression in adolescence and adulthood. Given that current theories on the pathways from childhood emotional abuse to subsequent depression (e.g., the hopelessness theory of depression and the stress generation theory of depression) are based on isolated investigations of researchers' interests rather than systematic empirical studies, this systematic review may contribute to a move to empirical research based on a more comprehensive theoretical model that incorporates the different potential psychological mediators and organizes them within a hierarchical multilevel theoretical framework.

In this model, childhood emotional abuse and various psychological mediators are considered to have direct and indirect effects on symptoms of depression (see Figure 3). Specifically, childhood emotional abuse is hypothesized to affect early schemas or attachment internal working models in childhood and/or adolescence, which in turn influence the development of broad and more specific personality dimensions, resulting in problems with emotional regulation and problems in person-environment exchanges (i.e., problems with salutogenesis), further expressed as (a) self-generated negative events, (b) heightened sensitivity to stressors, and (c) interpersonal problems in adolescence and adulthood.

In this integrated model, experiences of emotional abuse are thought to first affect early schemas or attachment internal working models in childhood and/or adolescence. 


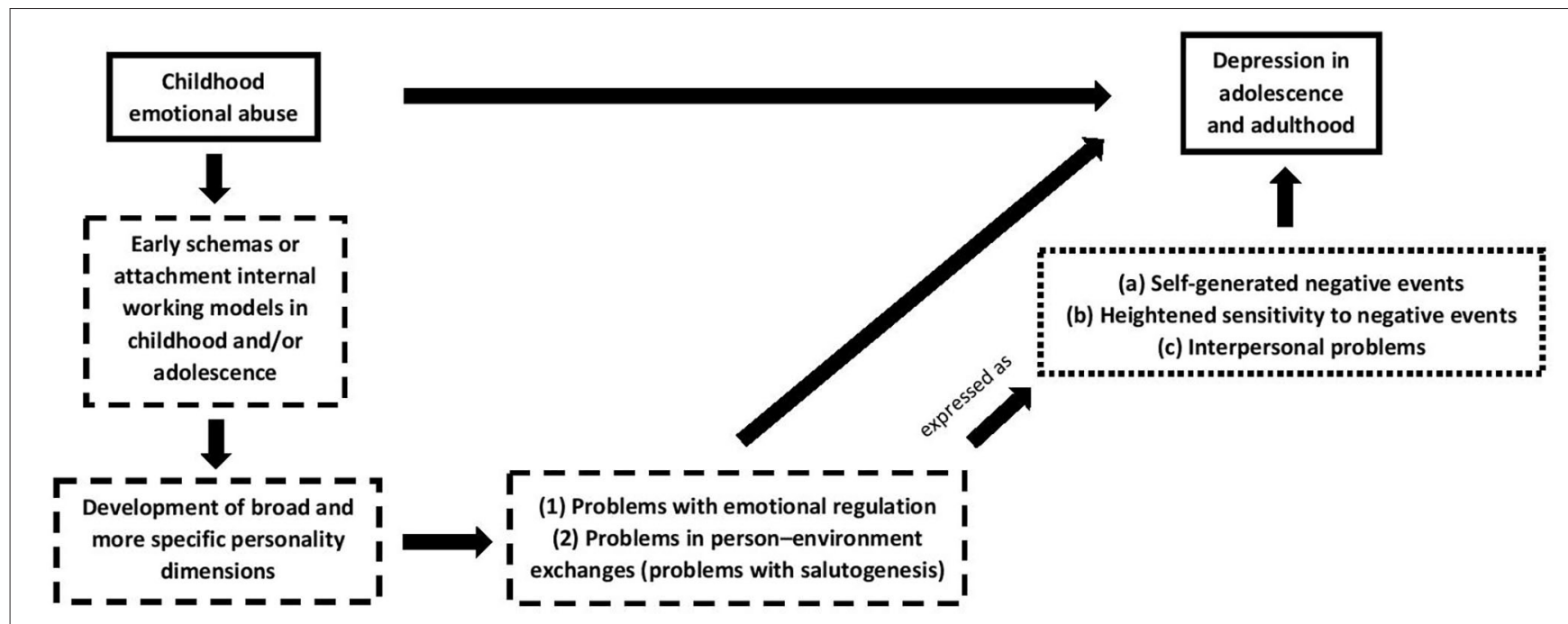

FIGURE 3 | A multilevel integrative model of the relationship between childhood emotional abuse and adult depression.

Studies included in the current review investigated the role of maladaptive schemas in the relation between emotional abuse and depression based on Young and colleagues' schema theory (105). Four studies addressed the pathways of particular schema themes that mediated the emotional abuse-depression link, including vulnerability to harm, self-sacrifice, social isolation, defectiveness and shame, disconnection and rejection, impaired autonomy, loneliness, and submission. It has been suggested that early maladaptive schemas might originate from unmet childhood needs for secure attachment (139). However, only two studies $(31,51)$ included in our review assessed attachment styles as a potential mediator, finding that insecure attachment styles prospectively mediated the association between a childhood history of emotional maltreatment and depressive symptoms in young adults, but were not significantly correlated with childhood emotional abuse or current depression in a small sample of community-dwelling older adults.

In this integrative theoretical model, we consider that early schemas and attachment internal working models in childhood and adolescence give rise to the development of broad and more specific personality dimensions (i.e., character tendencies and cognitive and emotion-related factors), resulting in problems with emotional regulation and problems in personenvironment exchanges (problems with salutogenesis). The schemas of vulnerability to harm and self-sacrifice appeared as mediators in the emotional abuse-depression link in our review. The schema of vulnerability to harm highlights fear and helplessness about the future as a lasting sequel of emotional abuse, which might consolidate into a more pervasive negative cognitive style (31). There was robust evidence for a cognitive vulnerability pathway linking childhood emotional abuse and later depression. Especially consistent evidence was found for hopelessness mediating the emotional abuse-depression link $(30,65,67)$. These findings fit with the hopelessness theory of depression developed by Abramson et al. (111), which postulates that chronic experiences of emotional abuse across different situations will induce hopelessness and lead to the development of an internal, stable, and global inferential style; this type of inference is generalized to other negative events, which accounts for subsequent increases in depressive symptoms. Gibb et al. (66) found that the relation between childhood emotional maltreatment and non-endogenous major depression was fully mediated by the presence vs. absence of negative cognitive styles; however, this was no longer the case when hopeless depression was controlled for. As such, it appeared that the etiological chain of the hopelessness theory accounted for only part of the emotional abuse-depression link. This assumption was also supported by Gibb et al. (67) who found that childhood emotional maltreatment led to negative attributions and inferences about specific experiences relating to emotional maltreatment, rather than cognitive vulnerability more generally.

In comparison, the schema of self-sacrifice refers to an excessive focus on others' desires and responses at the expense of one's own needs, so that individuals who are self-sacrificing typically believe that their own needs and emotions must be suppressed and inhibited (84). Hence, the schema of selfsacrifice might contribute to problems with emotional regulation as reflected in emotional inhibition and thought suppression, which are further demonstrated as experiential avoidance. Early maladaptive schemas refer not only to the content of one's thoughts but also to the repetitive and ruminative nature in which the person focuses on negative thoughts. Lumley and Harkness (89) found that tightly connected negative schema statements and loosely connected positive schema statements mediated the emotional abuse-depression link. This repetitive, ruminative nature of maladaptive schemas overlaps the cluster of emotion dysregulation in brooding rumination, consistent with the finding that brooding rumination was a mediator in the relation between early maladaptive schemas and symptoms of depression in adolescents (140). As such, the different clusters of mediators considered in the context of the present review 
can overlap significantly, and most probably interact with and reinforce each other.

Yet, Young et al. (105) have argued that early maladaptive schemas originate as the result of the interaction between early experiences and the child's temperament. The influence of emotional abuse on maladaptive schemas and cognitive vulnerabilities might be particularly strong when early experiences interact with specific temperament dimensions, such as high Neuroticism and low Extroversion (141). Consistent with this assumption, high Neuroticism, low Extroversion, and low Conscientiousness have not only been consistently linked to the onset of MDD (115) but have also been found to be a mediator in the emotional abuse-depression link (69). Calvete (62) examined temperament as a moderator in a 1-year follow-up to test the hypothesis that parental emotional abuse interacting with temperament predicts a worsening of maladaptive schemas. However, emotional abuse and temperament seemed not to be related in their study. Thus, a direction for future research is to clarify the mechanism through which specific temperamental diatheses interact with early emotional abuse in the ongoing development of specific schema themes, thereby resulting in vulnerability to depression.

Finally, problems with emotional regulation and problems in person-environment exchanges (problems with salutogenesis) are expressed as (a) self-generated negative events, (b) heightened sensitivity to stressors, and (c) interpersonal problems in adolescence and adulthood. Both Hankin (31) and Uhrlass and Gibb (82) reported that stress associated with negative life events served as the mechanism linking childhood emotional abuse to current depression symptoms. Regarding stressful negative events, it is important to distinguish between (a) occurrence of life stress, which may be in part self-generated, and (b) subjective perception of life events. Hankin (31) measured a prospective increase in the number of negative life events that typically occur for young adults, from school/achievement to interpersonal/romantic difficulties. Uhrlass and Gibb (82) focused on hassles rather than major negative life events (e.g., deaths or loss of job) in their study, and levels of negative life events were calculated by summing the number of hassles endorsed rather than the subjective impact ratings. Thus, both studies fall into the category of occurrence of life stress, and are consistent with Hammen's model where childhood emotional abuse may set the stage for self-generated negative events and depressive symptoms, as well as the elaborated cognitive vulnerability-transactional stress model of depression (142). Hankin (31) claimed that childhood emotional maltreatment does not appear to serve merely as an additional risk factor for depression; rather, it may contribute to the onset of an ongoing cycle of negative events and depression in adulthood.

In respect of the subjective perception of life events, individuals with a history of childhood emotional abuse are at greater risk of developing depression through the proximal factor of a negative cognitive style for depression, which noticeably overlaps the cluster of cognitive-personality variables. This subjective perception of life events is highly intertwined with heightened sensitivity to stressors. Post's (143) stress sensitization hypothesis proposes that a history of childhood maltreatment lowers the threshold of stress necessary to trigger depression onset, so that individuals with a history of childhood maltreatment are more reactive and responsive to stressors (144-146). In addition, parental emotional abuse is likely to increase one's negative appraisal of stressful life events, and this relationship might be mediated by affective temperaments (147). Although Hayashi et al. (69) found no correlation between negative life events and personality, Monroe and Harkness (145) suggested that people with a characteristically negative style of interpersonal interactions tend to have more stressful life events. Future research should examine whether temperament interacts with stressful negative life events when integrated into the same structural model to help elucidate the common and specific etiological factors for depression.

With regard to problems with emotional regulation being manifested as interpersonal problems in adolescence and adulthood, Barthel et al. (148) have argued that emotion regulation is directly linked with one's ability to effectively navigate the social world. Engagement in emotion regulation often occurs interpersonally with trusted others helping to regulate one's emotions (148). One study included in our review, by Christ et al. (32), identified two specific domains of interpersonal problems-cold/distant and domineering/controlling-as particularly important in explaining the emotional abuse-depression link. Children with a history of emotional abuse are likely to develop negative internal working models of the self and others as well as maladaptive schemas that interfere with social functioning as the child matures, leading to experiential avoidance and a lack of trust in others (149). This avoidance, as an emotion regulation strategy, in interpersonal relationships and social situations can be seen as a cold/distant interpersonal style. By contrast, the examination of a domineering/controlling interpersonal style in depression has received inconsistent results $(125,127)$. In addition to cold/distant and domineering/controlling interpersonal styles, other interpersonal problems, such as self-sacrificing, non-assertive, intrusive/needy, and overly accommodating, also theoretically relate to the emotional abuse-depression link. However, Sachs-Ericsson et al. (79) found no evidence of a dependent interpersonal style as a mediator in this link, although the dependent characteristic reflects extreme distress in relation to a strong need for approval and has been posited as a vulnerability marker for depression. Future studies are needed to examine which specific interpersonal styles stem from childhood emotional abuse, and to what extent these types of interpersonal problems may be associated with a greater risk of depression.

\section{Directions for Future Research}

Our findings support several potential psychological routes with relative consistency to adolescent and adult depression following a history of childhood emotional abuse (e.g., early maladaptive schemas, hopelessness, negative cognitive styles, brooding rumination, and overall emotion dysregulation). Further investigation is recommended to replicate the findings of those mediators that have been examined only a few times (e.g., personality traits, interpersonal styles). It is highly likely that considerable overlap exists between various mediating constructs and that several mediators may represent manifestations of 
other underlying processes (e.g., the important role of cognitive processes underlying the regulation of emotion). There is a lack of clarity regarding the extent to which these processes are relatively independent from each other, as well as their relative contribution to explaining risk for depression in those who have experienced childhood emotional abuse. Future research is needed to (a) compare the effect size of different mediators, (b) explore the interrelation between various mediators, (c) determine the degree of overlap, to disentangle the independent contribution of these different processes, and (d) elucidate whether certain types of experiences are particularly likely to trigger certain mechanisms. It is also possible that some of the psychological variables reviewed in the current study have a moderating role, as opposed to a mediating one, in the emotional abuse-depression link. For example, Hoppen and Chalder (24) found that romantic attachment avoidance and low self-esteem served as moderators, rather than mediators, in the relationship of childhood adversity with affective disorders. It is therefore unwarranted to assume that factors that do not appear to mediate the emotional abuse-depression link in this review do not play a role in the development of depression.

Current theories and evidence explaining the emotional abuse-depression link are largely built on isolated investigations based on researchers' interests and constrained by research funding and sample sizes, which may at times narrow findings at the expense of the bigger picture. To scrutinize whether psychological mediators function as a complex interrelated system in the emotional abuse-depression link, this review proposes a multilevel integrative model. Such an approach would help to move isolated focuses forward to empirical research based on a more comprehensive theoretical model that incorporates the different potential psychological mediators. This future work will require larger sample sizes, longitudinal designs, and more complex modeling techniques, allowing the robust appraisal of different pathways from childhood emotional abuse to subsequent depression.

Future research should also take resilience and protective factors into consideration. Not all emotionally maltreated children develop lifelong maladaptation. A focus on the individual differences between childhood emotional abuse victims can help us understand how some people overcome the emotional challenges of growing up in such environments. Studied protective factors for childhood maltreatment include attributional style (e.g., attribute abuse to external causes), early childhood secure attachment, and environmental factors, such as school and the presence of supportive relationships (150, 151). This line of research will help establish protective factors across the family system, community, school, and peer group to moderate the effects of parental emotional abuse.

Finally, future research should involve neurobiological processes related to identified psychological mechanisms in investigating the paths. Childhood adversity has been shown to be particularly detrimental, with long-lasting effects, during early stages of brain development (152). The causes of depression are most likely to be multifactorial, with interplay between genetic, neurobiological, psychological, and social factors. This line of future work will benefit from the integration of neurobiological and psychosocial approaches to illuminate the pathways that lead from childhood emotional abuse to depression in adolescence and adulthood.

\section{Limitations}

Findings should be considered in light of some limitations. Based on our quality assessment, more than half of the included studies $(n=18)$ were judged to be at high risk for selection bias, 13 studies did not control for the influence of potentially relevant confounders, such as age, gender, ethnicity, and socioeconomic status, and, importantly, eight studies were rated as weak in terms of the relative strength of the statistical mediation analysis. Given that the relationship between childhood maltreatment and later affective disorders has been found to be dependent on the age of exposure $(153,154)$, doses $(2,155)$, and severity $(20,156)$, a level of caution is required in the interpretation of the relative contribution of the mediators examined in the studies receiving weak ratings. In addition, more than twothird of the eligible studies $(n=24)$ were cross-sectional, where the relationship between the mediators and depressive symptoms is hard to disentangle as mediation is an inherently longitudinal process. Given that the quality of the studies might be impaired by the bidirectional relationship between risk factors for depression and depressive symptoms, the mediators identified in the cross-sectional studies should be accepted with caution.

An outstanding potential moderator that needs to be taken into consideration is the age of exposure. Twenty-six studies used adult samples (including one study investigating older participants), of which 11 used young adults recruited from universities, and eight studies used adolescent samples. Childhood emotional abuse might be more strongly predictive of depression onset in adolescence than in adulthood $(157,158)$, and the depressogenic effect of childhood maltreatment has also been shown to be stronger in adolescence than in adulthood (159). Considerable research has pointed to the dramatic increase in interpersonal stressful events and heightened sensitivity to social situations that occurs during adolescence as significant contributors to depressive symptoms. Affective processing (160) and top-down processes involved in understanding and identifying emotions are still developing during adolescence (161). Adolescence is a developmental period where individuals often experience their first depressive episode (162). The point prevalence of MDD in adolescence ranges from 3 to $8 \%$, with lifetime rates reaching $20 \%$ by the end of adolescence (163). La Rocque et al. (164) examined the moderating role of age in the relation of childhood maltreatment with sensitization to stressors that occurred just before episode onset. They found that adolescents (but not adults) with a history of maltreatment reported a lower level of severity of life events before episode onset than those without such a history; moreover, this relation was specific to emotional abuse, not physical or sexual abuse. As such, adolescents with a history of childhood emotional abuse may be more strongly sensitized to stressful life events that are crucial to triggering depression than adults with a similar history. Moreover, given that childhood maltreatment is closer in time to depression onset for adolescents who may still be living in an environment characterized by family discord and parental cruelty than for adults (165), childhood emotional abuse is likely to have 
a stronger etiological relation to depression onset in adolescence than in adulthood.

Notably, a majority of studies have samples composed of participants who are primarily Caucasian, women, university students, high-risk groups, low-income groups, or some combination thereof. One-third of included studies $(n=11)$ were conducted with young adult participants recruited from universities, who represent a fairly high-functioning sample with relatively homogeneous backgrounds. As such, the findings may be limited in generalizability to other populations. Nonetheless, well-educated young adult women have been shown to be an important high-risk group for developing depression (166). Not only are women twice as likely as men to experience MDD (167); a recent survey across eight countries demonstrated depressive disorder to be the most common mental disorder in firstyear university students, with a 12 -months prevalence of $18.5 \%$ (168). In addition, only a few mediators in eligible studies (e.g., early maladaptive schemas) have been examined in both clinical samples and non-clinical community samples. It will be important to examine whether the current findings of various mediators are replicated in both clinical and general samples and in more ethnically diverse samples.

Another limitation that has been repeatedly pointed out in the literature is the reliance on retrospective recall of childhood emotional abuse. There is a possibility of age and memory biases during affective episodes related to childhood maltreatment. As mentioned, some studies have reported that childhood maltreatment affected adolescents and young adults more strongly than middle-aged and older adults (164). However, some researchers found that childhood maltreatment had certain effects on older adults (169). Recent studies suggest the recall and mood effect can be negligible and retrospective reports can be highly consistent with prospective designs (170-172). In addition, as studies using a crosssectional design do not allow any firm conclusions to be made regarding the direction of the present relationship between childhood emotional abuse, potential mediating factors, and later depression, in order to arrive at more solid ground it would be necessary for each potential mediator to be replicated in a longitudinal design.

There were also some limitations to the review itself. While our search strategy was sensitive and incorporated the screening of references and citations of eligible studies, included articles were restricted to those published in peer-reviewed Englishlanguage journals, which may have resulted in relevant evidence being overlooked. The exclusion of unpublished reports, including master's and doctoral theses, may impact on the current findings, as published sources may overrepresent outcomes where significant mediation via psychological processes is observed. However, our search strategy did not include the terms "mediator" or "mechanism" and therefore we were more likely to retrieve studies reporting diverse, rather than only positive, findings. Future evidence syntheses may include more thorough examination of gray literature or estimate the extent to which publication bias and other selection biases might affect the findings of the review. Despite these limitations, the current review represents an initial step in building a comprehensive multilevel theoretical framework to guide future more integrative research efforts in this field.

\section{Clinical Implications}

Childhood maltreatment has been linked to treatment resistance in affective disorders $(9,173,174)$ as well as greater functional impairment (175). Some researchers have claimed that childhood maltreatment-related affective disorders should be considered a clinically distinct subtype (176). Our results are in line with numerous studies arguing that early abusive experiences should be routinely assessed in clinical practice, and it is important for clinicians to recognize and assess any core dysfunctional beliefs. In comparison with other more "tangible" forms of maltreatment, a history of emotional abuse may be more elusive and subtle to detect in intervention. The very nature of emotional abuse contributes it the possibility that it may "hide in plain sight" from practitioners and help to explain the fact that few interventions exist that explicitly target a history of parental emotional abuse (43). Our findings represent potentially valuable intervention targets that clinicians should consider while developing prevention and treatment plans for victims of childhood emotional abuse who have distressing symptoms of depression. As these mediators are very likely to function as a complex interrelated system, clinical work should include work on identified pathways, with the awareness that addressing each one may also positively impact on the others.

Cognitive and emotion-related factors, sensitivity to stressors, emotion dysregulation, and interpersonal style are often explicitly targeted in therapeutic and preventive interventions that are already recommended for depression, such as cognitive-behavioral therapy, interpersonal psychotherapy, emotion-focused therapy, acceptance-based psychotherapeutic interventions, such as dialectical behavior therapy and acceptance and commitment therapy, as well as psychodynamic and mindfulness-based approaches. Schema therapy, which includes key strategies to modify early maladaptive schemas, such as emotional imagery, interpersonal techniques, cognitive restructuring, and self-empowerment exercises, would be helpful for victims of emotional abuse with chronic dysfunctional schemas. This review highlights the importance of personalized therapy plans, as well as preventive and public health interventions aimed at individuals with a history of childhood emotional abuse.

\section{CONCLUSIONS}

The current systematic review demonstrates several potential mechanisms that might be involved in the relation between childhood emotional abuse and adolescent and adult depression. These psychological mediators fall into five clusters: (1) early maladaptive schemas, (2) cognitive-personality variables, (3) emotion dysregulation, (4) interpersonal styles, and (5) stressful negative events. However, the choice of examined variables to date has been limited and selective, suggesting that the conceptual framework guiding research in this field needs an integrative developmental cascade approach. Potentially significant elements of the emotional abuse-depression link may 
remain invisible as relevant variables have not been sufficiently examined. For example, we found no study examining either guilt or anger/aggression as a mediator, although they are two central concepts in the psychodynamic understanding of depression. We also hope to see more research on resilience and protective factors that might buffer children who are at risk for emotional and behavioral maladjustments. Most importantly, our postulated multilevel integrative model aims to inspire future research that directly addresses a coherent and integrated developmental theory or model in the emotional abuse-depression link. Ultimately, we hope to see a shift from isolated focuses in this area toward empirical research based on a more comprehensive theoretical model that incorporates the different potential psychological mediators and organizes them within a hierarchical multilevel theoretical framework.

\section{REFERENCES}

1. Bifulco A, Schimmenti A, Moran P, Jacobs C, Bunn A, Rusu AC. Problem parental care and teenage deliberate self-harm in young community adults. Bull Menninger Clini. (2014) 78:95-114. doi: 10.1521/bumc.2014.78.2.95

2. Chapman DP, Whitfield CL, Felitti VJ, Dube SR, Edwards VJ, Anda RF. Adverse childhood experiences and the risk of depressive disorders in adulthood. J Affect Disord. (2004) 82:217-25. doi: 10.1016/j.jad.2003.12.013

3. Rehan W, Antfolk J, Johansson A, Jern P, Santtila P. Experiences of severe childhood maltreatment, depression, anxiety and alcohol abuse among adults in Finland. PLoS ONE. (2017) 12:e0177252. doi: 10.1371/journal.pone.0177252

4. Moretti MM, Craig SG. Maternal versus paternal physical and emotional abuse, affect regulation and risk for depression from adolescence to early adulthood. Child Abuse Neglect. (2013) 37:4-13. doi: 10.1016/j.chiabu.2012.09.015

5. Widom CS, DuMont K, Czaja SJ. A prospective investigation of major depressive disorder and comorbidity in abused and neglected children grown up. Arch Gen Psychiatry. (2007) 64:49-56. doi: 10.1001/archpsyc.64.1.49

6. Suija K, Aluoja A, Kalda R, Maaroos HI. Factors associated with recurrent depression: a prospective study in family practice. Fam Pract. (2011) 28:2228. doi: 10.1093/fampra/cmq076

7. Infurna MR, Reichl C, Parzer P, Schimmenti A, Bifulco A, Kaess M. Associations between depression and specific childhood experiences of abuse and neglect: a meta-analysis. J Affect Disord. (2016) 190:47-55. doi: 10.1016/j.jad.2015.09.006

8. Lindert J, von Ehrenstein OS, Grashow R, Gal G, Braehler E, Weisskopf MG. Sexual and physical abuse in childhood is associated with depression and anxiety over the life course: systematic review and meta-analysis. Int J Public Health. (2014) 59:359-72. doi: 10.1007/s00038-013-0519-5

9. Nanni V, Uher R, Danese A. Childhood maltreatment predicts unfavorable course of illness and treatment outcome in depression: a meta-analysis. Am J Psychiatry. (2012) 169:141-51. doi: 10.1176/appi.ajp.2011.11020335

10. Nelson J, Klumparendt A, Doebler P, Ehring T. Childhood maltreatment and characteristics of adult depression: meta-analysis. Br J Psychiatry. (2017) 210:96-104. doi: 10.1192/bjp.bp.115.180752

11. Cutajar MC, Mullen PE, Ogloff JR, Thomas SD, Wells DL, Spataro J. Psychopathology in a large cohort of sexually abused children followed up to 43 years. Child Abuse Neglect. (2010) 34:813-22. doi: 10.1016/j.chiabu.2010.04.004

12. Fergusson DM, Boden JM, Horwood LJ. Exposure to childhood sexual and physical abuse and adjustment in early adulthood. Child Abuse Neglect. (2008) 32:607-19. doi: 10.1016/j.chiabu.2006.12.018

13. Liu RT, Jager-Hyman S, Wagner CA, Alloy LB, Gibb BE. Number of childhood abuse perpetrators and the occurrence of depressive episodes in adulthood. Child Abuse Neglect. (2012) 36:323-32. doi: 10.1016/j.chiabu.2011.11.007

\section{DATA AVAILABILITY STATEMENT}

The original contributions presented in the study are included in the article/supplementary material, further inquiries can be directed to the corresponding author/s.

\section{AUTHOR CONTRIBUTIONS}

EL conducted the systematic search and data extraction. Uncertainties during this period were resolved through discussions among the authors. The initial writing up was performed by EL under the supervision of PL and NM. All authors contributed to the manuscript writing and further elaboration and approved the final manuscript.

14. Kendler KS, Bulik CM, Silberg J, Hettema JM, Myers J, Prescott CA. Childhood sexual abuse and adult psychiatric and substance use disorders in women: an epidemiological and Cotwin control analysis. Arch Gen Psychiatry. (2000) 57:953-9. doi: 10.1001/archpsyc.57.10.953

15. Liu RT, Alloy LB, Abramson LY, Iacoviello BM, Whitehouse WG. Emotional maltreatment and depression: prospective prediction of depressive episodes. Depress Anxiety. (2009) 26:174-81. doi: 10.1002/da.20545

16. Martins CMS, Baes CVW, de Carvalho Tofoli SM, Juruena MF. Emotional abuse in childhood is a differential factor for the development of depression in adults. J Nerv Ment Dis. (2014) 202:774-82. doi: 10.1097/NMD.0000000000000202

17. Paul E, Eckenrode J. Childhood psychological maltreatment subtypes and adolescent depressive symptoms. Child Abuse Neglect. (2015) 47:38-47. doi: 10.1016/j.chiabu.2015.05.018

18. Goldman J, Salus MK, Wolcott D, Kennedy KY. What is child maltreatment? In: Goldman J, editor. A Coordinated Response to Child Abuse and Neglect: The Foundation for Practice. Washington, DC: US Department of Health and Human Services, Administration for Children and Families, Administration on Children, Youth and Families, Children's Bureau, Office on Child Abuse and Neglect (2003). p. 19.

19. Webb M, Heisler D, Call S, Chickering SA, Colburn TA. Shame, guilt, symptoms of depression, reported history of psychological maltreatment. Child Abuse Neglect. (2007) 31:1143-53. doi: 10.1016/j.chiabu.2007. 09.003

20. Norman RE, Byambaa M, De R, Butchart A, Scott J, Vos T. The longterm health consequences of child physical abuse, emotional abuse, neglect: a systematic review and meta-analysis. PLoS Med. (2012) 9:e1001349. doi: 10.1371/journal.pmed.1001349

21. O'Mahen HA, Karl A, Moberly N, Fedock G. The association between childhood maltreatment and emotion regulation: two different mechanisms contributing to depression? J Affect Disord. (2015) 174:287-95. doi: 10.1016/j.jad.2014.11.028

22. Hart SN, Glaser D. Psychological maltreatment-maltreatment of the mind: a catalyst for advancing child protection toward proactive primary prevention and promotion of personal well-being. Child Abuse Neglect. (2011) 35:758-66. doi: 10.1016/j.chiabu.2011.06.002

23. Trocmé N, Fallon B, MacLaurin B, Chamberland C, Chabot M, Esposito T. Shifting definitions of emotional maltreatment: an analysis child welfare investigation laws and practices in Canada. Child Abuse Neglect. (2011) 35:831-40. doi: 10.1016/j.chiabu.2011.05.015

24. Hoppen TH, Chalder T. Childhood adversity as a transdiagnostic risk factor for affective disorders in adulthood: a systematic review focusing on biopsychosocial moderating and mediating variables. Clin Psychol Rev. (2018) 65:81-151. doi: 10.1016/j.cpr.2018.08.002

25. Antypa N, Van der Does A. Serotonin transporter gene, childhood emotional abuse and cognitive vulnerability to depression. Genes Brain Behav. (2010) 9:615-20. doi: 10.1111/j.1601-183X.2010.00593.x 
26. Shadrina M, Bondarenko EA, Slominsky PA. Genetics factors in major depression disease. Front Psychiatry. (2018) 9:334. doi: $10.3389 /$ fpsyt.2018.00334

27. Keller J, Gomez R, Williams G, Lembke A, Lazzeroni L, Murphy GM, et al. HPA axis in major depression: cortisol, clinical symptomatology and genetic variation predict cognition. Mol Psychiatry. (2017) 22:527-36. doi: $10.1038 / \mathrm{mp} .2016 .120$

28. Lumley MN, Harkness KL. Specificity in the relations among childhood adversity, early maladaptive schemas, symptom profiles in adolescent depression. Cogn Ther Res. (2007) 31:639-57. doi: 10.1007/s10608-006-9100-3

29. Crow T, Cross D, Powers A, Bradley B. Emotion dysregulation as a mediator between childhood emotional abuse and current depression in a lowincome African-American sample. Child Abuse Neglect. (2014) 38:1590-8. doi: 10.1016/j.chiabu.2014.05.015

30. Courtney EA, Kushwaha M, Johnson JG. Childhood emotional abuse and risk for hopelessness and depressive symptoms during adolescence. J Emot Abuse. (2008) 8:281-98. doi: 10.1080/10926790802262572

31. Hankin BL. Childhood maltreatment and psychopathology: prospective tests of attachment, cognitive vulnerability, stress as mediating processes. Cogn Ther Res. (2005) 29:645-71. doi: 10.1007/s10608-005-9631-z

32. Christ C, de Waal MM, Dekker JJ, van Kuijk I, van Schaik DJ, Kikkert MJ, et al. Linking childhood emotional abuse and depressive symptoms: the role of emotion dysregulation and interpersonal problems. PLOS ONE. (2019) 14:e0211882. doi: 10.1371/journal.pone.0211882

33. Braithwaite EC, O’Connor RM, Degli-Esposti M, Luke N, Bowes L. Modifiable predictors of depression following childhood maltreatment: a systematic review and meta-analysis. Transl Psychiatry. (2017) 7:e1162. doi: $10.1038 /$ tp.2017.140

34. Sperry DM, Widom CS. Child abuse and neglect, social support, psychopathology in adulthood: a prospective investigation. Child Abuse Neglect. (2013) 37:415-25. doi: 10.1016/j.chiabu.2013.02.006

35. Salazar AM, Keller TE, Courtney ME. Understanding social support's role in the relationship between maltreatment and depression in youth with foster care experience. Child Maltreat. (2011) 16:102-13. doi: 10.1177/1077559511402985

36. Paredes PP, Calvete E. Cognitive vulnerabilities as mediators between emotional abuse and depressive symptoms. J Abnormal Child Psychol. (2014) 42:743-53. doi: 10.1007/s10802-013-9828-7

37. Brensilver M, Negriff S, Mennen FE, Trickett PK. Longitudinal relations between depressive symptoms and externalizing behaviour in adolescence: moderating effects of maltreatment experience and gender. J Clin Child Adolesc Psychol. (2011) 40:607-17. doi: 10.1080/15374416.2011.581618

38. Brody GH, Yu T, Beach SR, Kogan SM, Windle M, Philibert RA. Harsh parenting and adolescent health: a longitudinal analysis with genetic moderation. Health Psychol. (2014) 33:401. doi: 10.1037/a0032686

39. Fritz J, de Graaff AM, Caisley H, Van Harmelen AL, Wilkinson PO. A systematic review of amenable resilience factors that moderate and/or mediate the relationship between childhood adversity and mental health in young people. Front Psychiatry. (2018) 9:230. doi: 10.3389/fpsyt.2018.00230

40. Aafjes-van Doorn K, Kamsteeg C, Silberschatz G. Cognitive mediators of the relationship between adverse childhood experiences and adult psychopathology: a systematic review. Dev Psychopathol. (2019) 32:1017-29. doi: 10.1017/S0954579419001317

41. Wolfe DA, McIsaac C. Distinguishing between poor/dysfunctional parenting and child emotional maltreatment. Child Abuse Neglect. (2011) 35:802-13. doi: 10.1016/j.chiabu.2010.12.009

42. Trickett PK, Mennen FE, Kim K, Sang J. Emotional abuse in a sample of multiply maltreated, urban young adolescents: issues of definition and identification. Child Abuse Neglect. (2009) 33:27-35. doi: 10.1016/j.chiabu.2008.12.003

43. Spinazzola J, Hodgdon H, Liang LJ, Ford JD, Layne CM, Pynoos R, et al. Unseen wounds: the contribution of psychological maltreatment to child and adolescent mental health and risk outcomes. Psychol Trauma. (2014) 6:S18-28. doi: 10.1037/a0037766

44. Hart SN, Brassard MR, Karlson HC. Psychological maltreatment. In: Briere JN, Berliner LA, Bulkley J, Jenny CA, Reid TA, editors. The APSAC Handbook on Child Maltreatment. Thousand Oaks, CA: Sage (1996). p. 72-89.
45. Layne CM, Briggs-King E, Courtois C. Introduction to the special section: unpacking risk factor caravans across development: findings from the NCTSN core data set. Psychol Trauma. (2014) 6:S1-8. doi: 10.1037/a0037768

46. National Child Traumatic Stress Network. National Child Traumatic Stress Network Empirically Supported Treatments and Promising Practices. (2013). Retrieved from: http://www.nctsn.org/resources/topics/ treatments- thatwork/promising-practices

47. Vachon DD, Krueger RF, Rogosch FA, Cicchetti D. Different forms of child maltreatment have comparable consequences among children from low-income families. JAMA Psychiatry. (2015) 72:1135-42. doi: 10.1001/jamapsychiatry.2015.1792

48. Chamberland C, Laporte L, Lavergne C, Tourigny M, Mayer M, Wright J, et al. Psychological maltreatment of children reported to youth protective services: a situation of grave concern. J Emot Abuse. (2005) 5:65-94. doi: 10.1300/J135v05n01_03

49. Liberati A, Altman DG, Tetzlaff J, Mulrow C, Gotzsche et al. Research methods and reporting. Br Med J. (2009) 339:a2201. doi: 10.1136/bmj.b2700

50. Moher D, Liberati A, Tetzlaff J, Altman DG. Preferred reporting items for systematic reviews and meta-analyses: the PRISMA statement. Ann Intern Med. (2009) 51:264-69. doi: 10.7326/0003-4819-151-4-200908180-00135

51. Van Assche L, Van de Ven L, Vandenbulcke M, Luyten P. Ghosts from the past? The association between childhood interpersonal trauma, attachment and anxiety and depression in late life. Aging Ment Health. (2019) 24:898905. doi: 10.1080/13607863.2019.1571017

52. Khosravani V, Messman-Moore TL, Mohammadzadeh A, Ghorbani F, Amirinezhad A. Effects of childhood emotional maltreatment on depressive symptoms through emotion dysregulation in treatment-seeking patients with heroin-dependence. J Affect Disord. (2019) 256:448-57. doi: 10.1016/j.jad.2019.06.021

53. Ross ND, Kaminski PL, Herrington R. From childhood emotional maltreatment to depressive symptoms in adulthood: the roles of self-compassion and shame. Child Abuse Neglect. (2019) 92:32-42. doi: 10.1016/j.chiabu.2019.03.016

54. Li ET, Carracher E, Bird T. Linking childhood emotional abuse and adult depressive symptoms: the role of mentalizing incapacity. Child Abuse Neglect. (2020) 99:104253. doi: 10.1016/j.chiabu.2019.104253

55. Harvey AG, Watkins E, editors. Attention. In: Cognitive Behavioural Processes Across Psychological Disorders: A Transdiagnostic Approach to Research and Treatment. Oxford: Oxford University Press (2004). p. 65-6.

56. Gibb BE, Alloy LB. A prospective test of the hopelessness theory of depression in children. J Clin Child Adolesc Psychol. (2006) 35:264-74. doi: 10.1207/s15374424jccp3502_10

57. Jardim GBG, von Gunten A, da Silva Filho IG, Ziegelmann PK, Bumaguin DB, Nogueira EL, et al. Relationship between childhood maltreatment and geriatric depression: the mediator effect of personality traits. Int Psychogeriatr. (2019) 31:1759-67. doi: 10.1017/S1041610219 000073

58. Wong AE, Dirghangi SR, Hart SR. Self-concept clarity mediates the effects of adverse childhood experiences on adult suicide behavior, depression, loneliness, perceived stress, life distress. Self Identity. (2019) 18:247-66. doi: 10.1080/15298868.2018.1439096

59. Hopfinger L, Berking M, Bockting CL, Ebert DD. Emotion regulation mediates the effect of childhood trauma on depression. J Affect Disord. (2016) 198:189-97. doi: 10.1016/j.jad.2016.03.050

60. Schierholz A, Krüger A, Barenbrügge J, Ehring T. What mediates the link between childhood maltreatment and depression? The role of emotion dysregulation, attachment, attributional style. Eur J Psychotraumatol. (2016) 7:32652. doi: 10.3402/ejpt.v7.32652

61. Alloy LB, Abramson LY, Smith JM, Gibb BE, Neeren AM. Role of parenting and maltreatment histories in unipolar and bipolar mood disorders: mediation by cognitive vulnerability to depression. Clin Child Fam Psychol Rev. (2006) 9:23-64. doi: 10.1007/s10567-006-0002-4

62. Calvete E. Emotional abuse as a predictor of early maladaptive schemas in adolescents: contributions to the development of depressive and social anxiety symptoms. Child Abuse Neglect. (2014) 38:735-46. doi: 10.1016/j.chiabu.2013.10.014

63. Carvalho Fernando S, Beblo T, Schlosser N, Terfehr K, Otte C, Löwe B, et al. The impact of self-reported childhood trauma on emotion regulation 
in borderline personality disorder and major depression. J Trauma Dissoc. (2014) 15:384-401. doi: 10.1080/15299732.2013.863262

64. Coates AA, Messman-Moore TL. A structural model of mechanisms predicting depressive symptoms in women following childhood psychological maltreatment. Child Abuse Neglect. (2014) 38:103-13. doi: 10.1016/j.chiabu.2013.10.005

65. Courtney EA, Johnson JG, Alloy LB. Associations of childhood maltreatment with hopelessness and depression among adolescent primary care patients. Int J Cogn Ther. (2008) 1:4-17. doi: 10.1521/ijct.2008.1.1.4

66. Gibb BE, Alloy LB, Abramson LY, Rose DT, Whitehouse WG, Donovan $\mathrm{P}$, et al. History of childhood maltreatment, negative cognitive styles, episodes of depression in adulthood. Cogn Ther Res. (2001) 25:425-46. doi: 10.1023/A:1005586519986

67. Gibb BE, Alloy LB, Abramson LY, Marx B. Childhood maltreatment and maltreatment-specific inferences: a test of rose and Abramson's (1992) extension of the hopelessness theory. Cogn Emot. (2003) 17:917-31. doi: 10.1080/02699930244000237

68. Baron MR, Kenny DA. The moderator-mediator variable distinction in social psychological research: conceptual, strategic, statistical considerations. J Pers Soc Psychol. (1986) 51:1173-82. doi: 10.1037/0022-3514.51.6.1173

69. Hayashi Y, Okamoto Y, Takagaki K, Okada G, Toki S, Inoue T, et al. Direct and indirect influences of childhood abuse on depression symptoms in patients with major depressive disorder. BMC Psychiatry. (2015) 15:244. doi: $10.1186 / \mathrm{s} 12888-015-0636-1$

70. Jessar AJ, Hamilton JL, Flynn M, Abramson LY, Alloy LB. Emotional clarity as a mechanism linking emotional neglect and depressive symptoms during early adolescence. J Early Adolesc. (2017) 37:414-32. doi: $10.1177 / 0272431615609157$

71. Kaysen D, Scher CD, Mastnak J, Resick P. Cognitive mediation of childhood maltreatment and adult depression in recent crime victims. Behav Ther. (2005) 36:235-44. doi: 10.1016/S0005-7894(05)80072-3

72. Krause ED, Mendelson T, Lynch TR. Childhood emotional invalidation and adult psychological distress: the mediating role of emotional inhibition. Child Abuse Neglect. (2003) 27:199-213. doi: 10.1016/S0145-2134(02) 00536-7

73. Preacher KJ, Hayes AF. SPSS and SAS procedures for estimating indirect effects in simple mediation models. Behav Res Methods Instruments Comput. (2004) 36:717-31. doi: 10.3758/BF03206553

74. Maciejewski P, Mazure K. Fear of criticism and rejection mediates an association between childhood emotional abuse and adult onset of major depression. Cogn Ther Res. (2006) 30:105-22. doi: 10.1007/s10608-006-9012-2

75. Østefjells T, Lystad JU, Berg AO, Hagen R, Loewy R, Sandvik L, et al. Metacognitive beliefs mediate the effect of emotional abuse on depressive and psychotic symptoms in severe mental disorders. Psychol Med. (2017) 47:2323-33. doi: 10.1017/S0033291717000848

76. Raes F, Hermans D. On the mediating role of subtypes of rumination in the relationship between childhood emotional abuse and depressed mood: brooding versus reflection. Depress Anxiety. (2008) 25:1067-70. doi: $10.1002 / \mathrm{da} .20447$

77. Rafi MA, Adibsereshki N, Aval MH. The mediating role of early maladaptive schemas in the relationships of emotional maltreatment and emotional disorders. Eur J Ment Health. (2017) 12:104-15. doi: 10.5708/EJMH.12.2017.1.7

78. Reddy MK, Pickett SM, Orcutt HK. Experiential avoidance as a mediator in the relationship between childhood psychological abuse and current mental health symptoms in college students. J Emot Abuse. (2006) 6:67-85. doi: 10.1300/J135v06n01_04

79. Sachs-Ericsson N, Verona E, Joiner T, Preacher KJ. Parental verbal abuse and the mediating role of self-criticism in adult internalizing disorders. J Affect Disord. (2006) 93:71-8. doi: 10.1016/j.jad.2006.02.014

80. Schulz P, Beblo T, Ribbert H, Kater L, Spannhorst S, Driessen M, et al. How is childhood emotional abuse related to major depression in adulthood? The role of personality and emotion acceptance. Child Abuse Neglect. (2017) 72:98-109. doi: 10.1016/j.chiabu.2017.07.022

81. Spasojevic J, Alloy LB. Who becomes a depressive ruminator? Developmental antecedents of ruminative response style. J Cogn Psychother. (2002) 16:405. doi: 10.1891/jcop.16.4.405.52529
82. Uhrlass D, Gibb B. Childhood emotional maltreatment and the stress generation model of depression. J Soc Clin Psychol. (2007) 26:119-30. doi: $10.1521 /$ jscp.2007.26.1.119

83. van Harmelen AL, de Jong PJ, Glashouwer KA, Spinhoven P, Penninx BW, Elzinga BM. Child abuse and negative explicit and automatic selfassociations: the cognitive scars of emotional maltreatment. Behav Res Ther. (2010) 48:486-94. doi: 10.1016/j.brat.2010.02.003

84. Wright MOD, Crawford E, Del Castillo D. Childhood emotional maltreatment and later psychological distress among college students: the mediating role of maladaptive schemas. Child Abuse Neglect. (2009) 33:5968. doi: 10.1016/j.chiabu.2008.12.007

85. Yigit I, Kiliç H, Yigit MG, Çelik C. Emotional and physical maltreatment, early maladaptive schemas, internalizing disorders in adolescents: a multigroup path model of clinical and non-clinical samples. Curr Psychol. (2018) 1-11. doi: 10.1007/s12144-018-0068-4

86. Thomas H. Quality Assessment Tool for Quantitative Studies: Effective Public Health Practice Project. (2003). Retrieved from: http://www.ephpp.ca/PDF/ Quality\%20Assessment\%20Tool_2010_2.pdf

87. Hayes AF. Beyond Baron and Kenny: statistical mediation analysis in the new millennium. Commun Monogr. (2009) 76:408-20. doi: 10.1080/03637750903310360

88. Hayes AF. PROCESS: A Versatile Computational Tool for Observed Variable Mediation, Moderation and Conditional Process Analysis Modelling (White Paper). (2012). Retrieved from: http://www.afhayes.com/public/process/ 2012.pdf

89. Lumley MN, Harkness KL. Childhood maltreatment and depressotypic cognitive organization. Cogn Ther Res. (2009) 33:511-22. doi: 10.1007/s10608-009-9257-7

90. MacKinnon DP, Fairchild AJ, Fritz MS. Mediation analysis. Annu Rev Psychol. (2007) 58:593-614. doi: 10.1146/annurev.psych.58.110405.085542

91. Bernstein DP, Fink L. Childhood Trauma Questionnaire: A Retrospective Self-Report: Manual. Harcourt Brace and Company (1998). Retrieved from: https://www.pearsonassessments.com/store/usassessments/en/Store/ Professional-Assessments/Personality-\%26-Biopsychosocial/ChildhoodTrauma-Questionnaire\%3A-A-Retrospective-Self-Report/p/100000446. html

92. Bernstein DP, Stein JA, Newcomb MD, Walker E, Pogge D, Ahluvalia $\mathrm{T}$, et al. Development and validation of a brief screening version of the childhood trauma questionnaire. Child Abuse Neglect. (2003) 27:169-90. doi: 10.1016/S0145-2134(02)00541-0

93. Valenzuela MJ, Sachdev P. Assessment of complex mental activity across the lifespan: development of the Lifetime of Experiences Questionnaire (LEQ). Psychol Med. (2007) 37:1015-26. doi: 10.1017/S003329170600938X

94. Bifulco A, Brown GW, Harris TO. Childhood experience of care and abuse (CECA): a retrospective interview measure. J Child Psychol Psychiatry. (1994) 35:1419-35. doi: 10.1111/j.1469-7610.1994.tb01284.x

95. Beck AT, Steer RA, Brown GK. Beck depression inventory-II. San Antonio. (1996) 78:490-8. doi: 10.1037/t00742-000

96. Beck AT, Ward CH, Mendelson M, Mock J, Erbaugh J. An inventory for measuring depression. Arch Gen Psychiatry. (1961) 4:561-71. doi: $10.1001 /$ archpsyc.1961.01710120031004

97. Radloff LS. The use of the center for epidemiologic studies depression scale in adolescents and young adults. J Youth Adolesc. (1991) 20:149-66. doi: 10.1007/BF01537606

98. Lovibond PF, Lovibond SH. The structure of negative emotional states: comparison of the depression anxiety stress scales (DASS) with the beck depression and anxiety inventories. Behav Res Ther. (1995) 33:335-43. doi: 10.1016/0005-7967(94)00075-U

99. Reidy J, Keogh E. Testing the discriminant and convergent validity of the mood and anxiety symptoms questionnaire using a British sample. Pers Individ Differ. (1997) 23:337-44.

100. Kovacs M, Beck AT. An empirical-clinical approach toward a definition of childhood depression. In: Schulterbrandt JG, Raskin A, editors. Depression in Childhood: Diagnosis, Treatment, Conceptual Models. New York, NY: Raven Press (1977). p. 1-25.

101. Steinberg M, Rounsaville B, Cicchetti DV. The structured clinical interview for DSM-III-R dissociative disorders: preliminary report on a new diagnostic instrument. Am J Psychiatry. (1990) 147:76-82. 
102. American Psychological Association, editor. Scope of the problem. In: Publication Manual of the American Psychological Association, 5th ed. Washington, DC: APA (2001). p. 1.

103. Kessler RC, Üstün TB. The world mental health (WMH) survey initiative version of the world health organization (WHO) composite international diagnostic interview (CIDI). Int J Methods Psychiatr Res. (2004) 13:93-121. doi: $10.1002 /$ mpr.168

104. Endicott J, Spitzer RL. A diagnostic interview: the schedule for affective disorders and schizophrenia. Arch Gen Psychiatry. (1978) 35:837-44. doi: 10.1001/archpsyc.1978.01770310043002

105. Young JE, Klosko JS, Weishaar ME, editors. Schema therapy: conceptual model. In: Schema Therapy: A Practitioner's Guide. New York, NY: Guilford Press (2003). p. 1-26.

106. Calvete E, Orue I, Hankin BL. Transactional relationships among cognitive vulnerabilities, stressors, depressive symptoms in adolescence. J Abnormal Child Psychol. (2013) 41:399-410. doi: 10.1007/s10802-012-9691-y

107. Digman JM. Personality structure: emergence of the five-factor model. Annu Rev Psychol. (1990) 41:417-40. doi: 10.1146/annurev.ps.41.020190.002221

108. Beck AT. Cognitive therapy of depression: new perspectives. In: Clayton PJ, Barnett JE, editors. Treatment of Depression: Old Controversies and New Approaches. New York, NY: Raven Press (1983). p. 265-90.

109. Bieling PJ, Beck AT, Brown GK. The sociotropy-autonomy scale: structure and implications. Cogn Ther Res. (2000) 24:763-80. doi: 10.1023/A:1005599714224

110. Davis DO, Freeman A. Overview of cognitive-behavioral therapy of personality disorders. In: Beck AT, Davis DD, Freeman A, editors. Cognitive Therapy of Personality Disorders. New York, NY: Guilford Publications (2015). p. 3-18.

111. Abramson LY, Metalsky GI, Alloy LB. Hopelessness depression: a theory-based subtype of depression. Psychol Rev. (1989) 96:358. doi: 10.1037/0033-295X.96.2.358

112. Calvete E. Integrating sociotropy, negative inferences and social stressors as explanations for the development of depression in adolescence: interactive and mediational mechanisms. Cogn Ther Res. (2011) 35:477-90. doi: 10.1007/s10608-010-9320-4

113. Calvete E, Villardón L, Estévez A. Attributional style and depressive symptoms in adolescents: an examination of the role of various indicators of cognitive vulnerability. Behav Res Ther. (2008) 46:944-53. doi: 10.1016/j.brat.2008.04.010

114. Cole DA, Ciesla JA, Dallaire DH, Jacquez FM, Pineda AQ, LaGrange B, et al. Emergence of attributional style and its relation to depressive symptoms. $J$ Abnormal Psychol. (2008) 117:16. doi: 10.1037/0021-843X.117.1.16

115. Rosellini AJ, Brown TA. The NEO five-factor inventory: latent structure and relationships with dimensions of anxiety and depressive disorders in a large clinical sample. Assessment. (2011) 18:27-38. doi: 10.1177/1073191110382848

116. DeYoung CG, Cicchetti D, Rogosch FA. Moderation of the association between childhood maltreatment and neuroticism by the corticotropinreleasing hormone receptor 1 gene. J Child Psychol Psychiatry. (2011) 52:898906. doi: 10.1111/j.1469-7610.2011.02404.x

117. Cohen LJ, Tanis T, Bhattacharjee R, Nesci C, Halmi W, Galynker I. Are there differential relationships between different types of childhood maltreatment and different types of adult personality pathology? Psychiatry Res. (2014) 215:192-201. doi: 10.1016/j.psychres.2013.10.036

118. Carr CP, Martins CMS, Stingel AM, Lemgruber VB, Juruena MF. The role of early life stress in adult psychiatric disorders: a systematic review according to childhood trauma subtypes. J Nerv Ment Dis. (2013) 201:100720. doi: 10.1097/NMD.0000000000000049

119. Iacoviello BM, Alloy LB, Abramson LY, Whitehouse WG, Hogan ME. The role of cluster $\mathrm{B}$ and $\mathrm{C}$ personality disturbance in the course of depression: a prospective study. J Pers Disord. (2007) 21:371-83. doi: 10.1521/pedi.2007.21.4.371

120. Robins CJ, Block P. Cognitive theories of depression viewed from a diathesis-stress perspective: evaluations of the models of beck and of Abramson, Seligman, Teasdale. Cogn Ther Res. (1989) 13:297-313. doi: 10.1007/BF01173475

121. Treynor W, Gonzalez R, Nolen-Hoeksema S. Rumination reconsidered: a psychometric analysis. Cogn Ther Res. (2003) 27:247-59. doi: 10.1023/A:1023910315561
122. Nolen-Hoeksema S, Wisco BE, Lyubomirsky S. Rethinking rumination. Perspect Psychol Sci. (2008) 3:400-24. doi: 10.1111/j.1745-6924.2008.00088.x

123. Cicchetti D, Toth SL. Child maltreatment. Annu Rev Clin Psychol. (2005) 1:409-38. doi: 10.1146/annurev.clinpsy.1.102803.144029

124. Colman RA, Widom CS. Childhood abuse and neglect and adult intimate relationships: a prospective study. Child Abuse Neglect. (2004) 28:1133-51. doi: 10.1016/j.chiabu.2004.02.005

125. Barrett MS, Barber JP. Interpersonal profiles in major depressive disorder. J Clin Psychol. (2007) 63:247-66. doi: 10.1002/jclp.20346

126. Liu RT. Childhood adversities and depression in adulthood: current findings and future directions. Clin Psychol. (2017) 24:140-53. doi: $10.1111 /$ cpsp. 12190

127. Huh HJ, Kim SY, Yu JJ, Chae JH. Childhood trauma and adult interpersonal relationship problems in patients with depression and anxiety disorders. Ann Gen Psychiatry. (2014) 13:26. doi: 10.1186/s12991-014-0026-y

128. Hammen C. Generation of stress in the course of unipolar depression. $J$ Abnormal Psychol. (1991) 100:555. doi: 10.1037/0021-843X.100.4.555

129. Hammen C. Cognitive, life stress, interpersonal approaches to a developmental psychopathology model of depression. Dev Psychopathol. (1992) 4:189-206. doi: 10.1017/S0954579400005630

130. Liu RT, Kraines MA, Massing-Schaffer M, Alloy LB. Rejection sensitivity and depression: mediation by stress generation. Psychiatry. (2014) 77:86-97. doi: 10.1521 /psyc.2014.77.1.86

131. Norouzi P. The comparison of neuropsychological function of emotional abused and nonabused female students (Master's thesis of Exceptional Children Psychology), Allame Tabatabae'i University, Tehran, Iran (2012).

132. Abela JR, Zuroff DC, Ho MHR, Adams P, Hankin BL. Excessive reassurance seeking, hassles, depressive symptoms in children of affectively ill parents: a multiwave longitudinal study. J Abnormal Child Psychol. (2006) 34:165-181. doi: 10.1007/s10802-005-9011-x

133. Watkins ER. Depressive rumination and co-morbidity: evidence for brooding as a transdiagnostic process. J Rational Emot Cogn Behav Ther. (2009) 27:160-75. doi: 10.1007/s10942-009-0098-9

134. Gibb BE, Hanley AJ. Depression and interpersonal stress generation in children: prospective impact on relational versus overt victimization. Int $J$ Cogn Ther. (2010) 3:358-67. doi: 10.1521/ijct.2010.3.4.358

135. Joormann J, Gotlib IH. Emotion regulation in depression: Relation to cognitive inhibition. Cogn Emotion. (2010) 24:281-98. doi: 10.1080/02699930903407948

136. Briere J, Jordan CE. Childhood maltreatment, intervening variables, adult psychological difficulties in women: an overview. Trauma Violence Abuse. (2009) 10:375-88. doi: 10.1177/1524838009339757

137. Hankin BL, Young JF, Abela JR, Smolen A, Jenness JL, Gulley LD, et al. Depression from childhood into late adolescence: influence of gender, development, genetic susceptibility, peer stress. J Abnormal Psychol. (2015) 124:803-16. doi: 10.1037/abn0000089

138. Hamilton JL, Shapero BG, Stange JP, Hamlat EJ, Abramson LY, Alloy LB. Emotional maltreatment, peer victimization, depressive versus anxiety symptoms during adolescence: hopelessness as a mediator. J Clin Child Adolesc Psychol. (2013) 42:332-47. doi: 10.1080/15374416.2013.777916

139. Simard V, Moss E, Pascuzzo K. Early maladaptive schemas and child and adult attachment: a 15-year longitudinal study. Psychol Psychother. (2011) 84:349-66. doi: 10.1111/j.2044-8341.2010.02009.x

140. Orue I, Calvete E, Padilla P. Brooding rumination as a mediator in the relation between early maladaptive schemas and symptoms of depression and social anxiety in adolescents. J Adolesc. (2014) 37:1281-91. doi: 10.1016/j.adolescence.2014.09.004

141. Epkins CC, Heckler DR. Integrating etiological models of social anxiety and depression in youth: evidence for a cumulative interpersonal risk model. Clin Child Fam Psychol Rev. (2011) 14:329-76. doi: 10.1007/s10567-011-0101-8

142. Hankin BL, Abramson LY. Development of gender differences in depression: an elaborated cognitive vulnerability-transactional stress theory. Psychol Bull. (2001) 127:773. doi: 10.1037/0033-2909.127.6.773

143. Post RM. Transduction of psychosocial stress into the neurobiology of recurrent affective disorder. Am J Psychiatry. (1992) 149:999. doi: 10.1176/ajp.149.8.999

144. Harkness KL, Bruce AE, Lumley MN. The role of childhood abuse and neglect in the sensitization to stressful life events in adolescent depression. J Abnormal Psychol. (2006) 115:730. doi: 10.1037/0021-843X.115.4.730 
145. Monroe SM, Harkness KL. Life stress, the "kindling" hypothesis, the recurrence of depression: considerations from a life stress perspective. Psychol Rev. (2005) 112:417. doi: 10.1037/0033-295X.112.2.417

146. Muscatell KA, Slavich GM, Monroe SM, Gotlib IH. Stressful life events, chronic difficulties, the symptoms of clinical depression. J Nerv Ment Dis. (2009) 197:154-60. doi: 10.1097/NMD.0b013e318199f77b

147. Nakai Y, Inoue T, Toda H, Toyomaki A, Nakato Y, Nakagawa S, et al. The influence of childhood abuse, adult stressful life events and temperaments on depressive symptoms in the nonclinical general adult population. J Affect Disord. (2014) 158:101-7. doi: 10.1016/j.jad.2014.02.004

148. Barthel A, Hay A, Doan S, Hofmann S. Interpersonal emotion regulation: a review of social and developmental components. Behav Change. (2018) 35:203-16. doi: 10.1017/bec.2018.19

149. Riggs S. Childhood emotional abuse and the attachment system across the life cycle: what theory and research tell US. J Aggress Maltreat Trauma. (2010) 19:5-51. doi: 10.1080/10926770903475968

150. Iwaniec D, Larkin E, Higgins S. Research review: risk and resilience in cases of emotional abuse. Child Fam Soc Work. (2006) 11:73-82. doi: 10.1111/j.1365-2206.2006.00398.x

151. Gariepy G, Honkaniemi H, Quesnel-Vallee A. Social support and protection from depression: systematic review of current findings in Western countries. Br J Psychiatry. (2016) 209:284-93. doi: 10.1192/bjp.bp.115.169094

152. Lupien SJ, McEwen BS, Gunnar MR, Heim C. Effects of stress throughout the lifespan on the brain, behaviour and cognition. Nat Rev Neurosci. (2009) 10:434-45. doi: 10.1038/nrn2639

153. Dunn EC, McLaughlin KA, Slopen N, Rosand J, Smoller JW. Developmental timing of child maltreatment and symptoms of depression and suicidal ideation in young adulthood: results from the national longitudinal study of adolescent health. Depress Anxiety. (2013) 30:955-64. doi: 10.1002/da.22102

154. Schoedl AF, Costa MCP, Mari JJ, Mello MF, Tyrka AR, Carpenter LL, et al. The clinical correlates of reported childhood sexual abuse: an association between age at trauma onset and severity of depression and PTSD in adults. J Child Sexual Abuse. (2010) 19:156-70. doi: 10.1080/10538711003615038

155. Anda RF, Felitti VJ, Bremner JD, Walker JD, Whitfield CH, Perry BD, et al. The enduring effects of abuse and related adverse experiences in childhood. Eur Arch Psychiatry Clin Neurosci. (2006) 256:174-86. doi: 10.1007/s00406-005-0624-4

156. Hovens JG, Wiersma JE, Giltay EJ, Van Oppen P, Spinhoven P, Penninx BW, et al. Childhood life events and childhood trauma in adult patients with depressive, anxiety and comorbid disorders vs. controls. Acta Psychiatr Scand. (2010) 122:66-74. doi: 10.1111/j.1600-0447.2009.01491.x

157. Brown GW, Craig TK, Harris TO, Handley RV, Harvey AL. Validity of retrospective measures of early maltreatment and depressive episodes using the childhood experience of care and abuse (CECA) instrument-A lifecourse study of adult chronic depression--2. J Affect Disord. (2007) 103:21724. doi: 10.1016/j.jad.2007.06.003

158. Gibb BE, Chelminski I, Zimmerman M. Childhood emotional, physical, sexual abuse, diagnoses of depressive and anxiety disorders in adult psychiatric outpatients. Depress Anxiety. (2007) 24:256-63. doi: $10.1002 /$ da. 20238

159. Hill J, Pickles A, Rollinson L, Davies R, Byatt M. Juvenile-versus adultonset depression: multiple differences imply different pathways. Psychol Med. (2004) 34:1483-93. doi: 10.1017/S0033291704002843

160. Burnett S, Sebastian C, Kadosh KC, Blakemore SJ. The social brain in adolescence: evidence from functional magnetic resonance imaging and behavioural studies. Neurosci Biobehav Rev. (2011) 35:1654-64. doi: 10.1016/j.neubiorev.2010.10.011

161. Casey BJ, Jones RM, Hare TA. The adolescent brain. Ann N Y Acad Sci. (2008) 1124:111-26. doi: 10.1196/annals.1440.010

162. Hankin BL, Abramson LY, Moffitt TE, Silva PA, McGee R, Angell KE. Development of depression from preadolescence to young adulthood: emerging gender differences in a 10-year longitudinal study. J Abnormal Psychol. (1998) 107:128. doi: 10.1037/0021-843X.107.1.128

163. Zalsman G, Brent DA, Weersing VR. Depressive disorders in childhood and adolescence: an overview: epidemiology, clinical manifestation and risk factors. Child Adolesc Psychiatric Clin. (2006) 15:827-41. doi: 10.1016/j.chc.2006.05.002

164. La Rocque CL, Harkness KL, Bagby RM. The differential relation of childhood maltreatment to stress sensitization in adolescent and young adult depression. J Adolesc. (2014) 37:871-82. doi: 10.1016/j.adolescence.2014.05.012

165. Shanahan L, Copeland WE, Costello EJ, Angold A. Child-, adolescentand young adult-onset depressions: differential risk factors in development? Psychol Med. (2011) 41:2265-74. doi: 10.1017/S0033291711000675

166. de Graaf R, Ten Have M, van Gool C, van Dorsselaer S. Prevalence of mental disorders and trends from 1996 to 2009. Results from the Netherlands mental health survey and incidence study-2. Soc Psychiatry Psychiatr Epidemiol. (2012) 47:203-13. doi: 10.1007/s00127-010-0334-8

167. Kessler RC. Epidemiology of women and depression. J Affect Disord. (2003) 74:5-13. doi: 10.1016/S0165-0327(02)00426-3

168. Auerbach RP, Mortier P, Bruffaerts R, Alonso J, Benjet C, Cuijpers P, et al. WHO world mental health surveys international college student project: prevalence and distribution of mental disorders. J Abnormal Psychol. (2018) 127:623-8. doi: 10.1037/abn0000362

169. Schulz A, Schmidt CO, Appel K, Mahler J, Spitzer C, Wingenfeld K, et al. Psychometric functioning, socio-demographic variability of childhood maltreatment in the general population and its effects of depression. Int $J$ Methods Psychiatric Res. (2014) 23:387-400. doi: 10.1002/mpr.1447

170. Pinto R, Correia L, Maia Â. Assessing the reliability of retrospective reports of adverse childhood experiences among adolescents with documented childhood maltreatment. J Fam Violence. (2014) 29:431-8. doi: 10.1007/s10896-014-9602-9

171. Fergusson DM, Horwood LJ, Boden JM. Structural equation modeling of repeated retrospective reports of childhood maltreatment. Int J Methods Psychiatric Res. (2011) 20:93-104. doi: 10.1002/mpr.337

172. Scott KM, McLaughlin KA, Smith DA, Ellis PM. Childhood maltreatment and DSM-IV adult mental disorders: comparison of prospective and retrospective findings. Br J Psychiatry. (2012) 200:469-75. doi: 10.1192/bjp.bp.111.103267

173. Tunnard C, Rane LJ, Wooderson SC, Markopoulou K, Poon L, Fekadu $A$, et al. The impact of childhood adversity on suicidality and clinical course in treatment-resistant depression. J Affect Disord. (2014) 152:122-30. doi: 10.1016/j.jad.2013.06.037

174. Angst J, Gamma A, Rössler W, Ajdacic V, Klein DN. Childhood adversity and chronicity of mood disorders. Eur Arch Psychiatry Clin Neurosci. (2011) 261:21-7. doi: 10.1007/s00406-010-0120-3

175. McLaughlin KA, Conron KJ, Koenen KC, Gilman SE. Childhood adversity, adult stressful life events, risk of past-year psychiatric disorder: a test of the stress sensitization hypothesis in a population-based sample of adults. Psychol Med. (2010) 40:1647-58. doi: 10.1017/S0033291709992121

176. Teicher MH, Samson JA. Childhood maltreatment and psychopathology: a case for ecophenotypic variants as clinically and neurobiologically distinct subtypes. Am J Psychiatry. (2013) 170:1114-33. doi: 10.1176/appi.ajp.2013.12070957

177. Shrout PE, Bolger N. Mediation in experimental and nonexperimental studies: new procedures and recommendations. Psychol Methods. (2002) 7:422-45. doi: 10.1037/1082-989X.7.4.422

178. MacKinnon DP, Lockwood CM, Hoffman JM, West SG, Sheets V. A comparison of methods to test mediation and other intervening variable effects. Psychol Methods. (2002) 7:83.

179. Varia R, Abidin RR, Dass P. Perceptions of abuse: effects on adult psychological and social adjustment. Child Abuse Neglect. (1996) 20:511-26.

180. Kent A, Waller G. The impact of childhood emotional abuse: An extension of the child abuse and trauma scale. Child Abuse Neglect. (1998) 22:393-9.

181. Hirschfeld RM, Klerman GL, Gouch HG, Barrett J, Korchin SJ, Chodoff P. A measure of interpersonal dependency. J Personal Assessment. (1977) $41: 610-8$.

Conflict of Interest: The authors declare that the research was conducted in the absence of any commercial or financial relationships that could be construed as a potential conflict of interest.

Copyright (C) 2020 Li, Luyten and Midgley. This is an open-access article distributed under the terms of the Creative Commons Attribution License (CC BY). The use, distribution or reproduction in other forums is permitted, provided the original author(s) and the copyright owner(s) are credited and that the original publication in this journal is cited, in accordance with accepted academic practice. No use, distribution or reproduction is permitted which does not comply with these terms. 